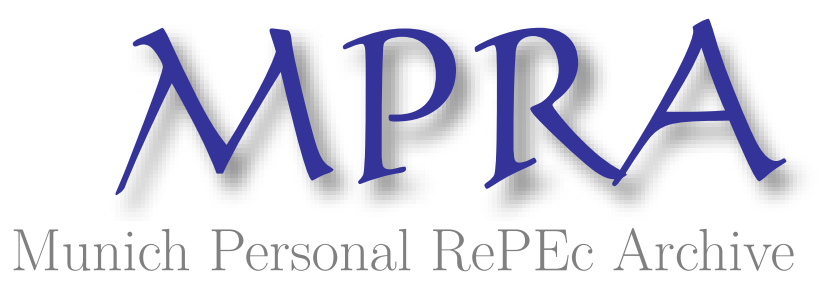

\title{
Random Marginal and Random Removal values
}

Calvo, Emilio

October 2006

Online at https://mpra.ub.uni-muenchen.de/142/

MPRA Paper No. 142, posted 25 Oct 2006 UTC 


\title{
Random Marginal and Random Removal values*
}

\author{
Emilio Calvo ${ }^{\dagger}$
}

October, 2006

\begin{abstract}
We propose two variations of the non-cooperative bargaining model for games in coalitional form, introduced by Hart and Mas-Colell (1996a). These strategic games implement, in the limit, two new NTU-values: The random marginal and the random removal values. The main characteristic of these proposals is that they always select a unique payoff allocation in NTU-games. The random marginal value coincides with the Consistent NTU-value (Maschler and Owen, 1989) for hyperplane games, and with the Shapley value for TU games (Shapley, 1953). The random removal coincides with the solidarity value (Novak and Radzik, 1994) in TU-games. In large games it is showed that, in the special class of market games, the random marginal coincides with the Shapley NTU-value (Shapley,1969), and that the random removal coincides with the equal split solution.
\end{abstract}

*This research has been supported by the DGES Ministerio de Educación y Ciencia under projects BEC2000-1429, BEC2000-0875and SEJ2004-07554. And from the Generalitat Valenciana under project GRUPOS04/13.

†Departamento de Análisis Económico and LINEEX. Universidad de Valencia. Avinguda dels Tarongers s/n. Edificio Departamental Oriental. 46022 Valencia. Spain. E-mail: Emilio.Calvo@uv.es 
JEL Classification: C71.

KEYWORDS: Shapley value; NTU-games; large market games.

\section{Introduction}

In this paper n-person cooperative games in coalitional form are considered.

When utility is transferable across players (TU-games) the most prominent solution concept is the Shapley (1953) value. It yields a unique payoff allocation for the players in the game. Shapley's original support for the value was axiomatic. Other relevant axiomatizations of the value are in Myerson (1980), and Hart and Mas-Colell (1989). Bargaining models that yield the value in the TU-case have also been proposed. Among these one should mention Gul (1989), Hart and Moore (1990), Hart and Mas-Colell (1996a), Winter (1994), and Pérez-Castrillo and Wettstein (2001).

When utility is not transferable (NTU-games), different ways to extend the value have been considered. The most relevant are by Harsanyi (1963), Shapley (1969) ${ }^{1}$, and Maschler and Owen $(1992)^{2}$. These three solutions were constructed in such a way that they coincide with the Nash (1950) solution for pure bargaining games and with the Shapley value for TU-games. Axiomatic support for these solutions have been carried out by Aumann (1985) for the Shapley NTU-solution, by Hart (1985) for the Harsanyi NTUsolution, and by de Clippel, Peters and Zank (2004) and by Hart (2005) for the Consistent NTU-solution. Bargaining games that support these solutions have been proposed for the Consistent NTU-solution in Hart and Mas-Colell (1996), and for the Shapley NTU-

\footnotetext{
${ }^{1}$ The Shapley NTU-value is also known as $\lambda$-transfer value.

${ }^{2}$ First introduced for hyperplane games in Maschler and Owen (1989), and also called the Consistent NTU-value.
} 
solution in Vidal-Puga $(2006)^{3}$.

This paper starts off with two aspects that should be taken into account in this value extension program: Single-valuedness and symmetry. Recall that both, the Shapley value and the Nash bargaining solution, satisfy symmetry and select single payoffs in TUgames, and pure bargaining games, respectively. Nevertheless, the Harsanyi, Shapley, and Consistent NTU-solutions do not guarantee uniqueness in the solution set.

Multiplicity of the outcomes is not a real problem if we interpret a solution from a predictive point of view. When a set is selected, that only means that the final outcome must belong to this solution set. This phenomenon happens in many other contexts, as in the Walrasian equilibria in competitive economic models, or in the Nash equilibria and many of its refinements for non-cooperative games. But from a normative point of view, this multiplicity in the solution set yields an inconsistency with respect to the symmetry axiom which belongs to the set of axioms that supports both the Shapley TU-value, and the Nash bargaining solution: It is easy to build examples ${ }^{4}$ of symmetric games for which asymmetric payoffs are selected by these three solutions. Where do these asymmetries come from? Perhaps a more detailed and explicit model of the bargaining rules that support each solution should be needed in order to understand the origin of these asymmetric payoffs.

From this strategic approach, the only relevant proposal is the bargaining procedure proposed by Hart and Mas-Colell (1996a). This model is an elegant and simple variation of the alternating offers method. It has the merit that it supports the Nash bargaining solution for pure bargaining games, the Shapley value for TU-games, and the Consistent solution for NTU-games. In brief, the model goes as follows:

\footnotetext{
${ }^{3}$ Only for the particular case in which the boundary of the grand coalition is a hyperplane.

${ }^{4}$ See Section 3 wherea two-person example is used extensively.
} 
Among the players ${ }^{5}$ still in the bargaining, a proposer is chosen randomly (with uniform distribution). The proposer makes a feasible offer. If the rest of the players agree, this offer is the final payoff. If any player rejects, with a prespecified probability $\rho(0 \leq \rho<1)$, a new proposer is chosen (among all of them) and the process is repeated again, and with probability $(1-\rho)$ the proposer drops out of the game (receiving zero) and we restart the bargaining process with the remaining players, and so on. The process starts initially with all players.

This bargaining procedure is a sequential, perfect information game, and it has a stationary subgame perfect equilibria. Moreover, when the probability $\rho$ goes to one, the solution payoffs associated to the equilibria converge to the consistent values. Moreover, in the same paper, some variations of this bargaining game are considered. And they obtain, in the authors' words, “...in particular, we allow for the possibility that players other than the proposer may be the victims of bargaining breakdown. However, we show that if the bargaining procedure yields the Shapley value in the TU-case, then necessarily the consistent value obtains in the NTU-case. Thus the consistent NTU-value is, according to our noncooperative approach, the appropriate generalization of the Shapley TU-value."

End of story?

First of all, note that this bargaining model does not yield a full support of the set of consistent payoff allocations, i.e. there are examples with consistent payoff allocations that cannot be approached (when $\rho \rightarrow 1$ ) with the payoffs associated to the stationary subgame perfect equilibria. This fact will be shown by the example discussed in Section 3: In this symmetric game there are three consistent payoffs, two of them asymmetric and only one symmetric. The asymmetric points are the only ones that can be approached

\footnotetext{
${ }^{5}$ From now on, we interpret players in a game as agents with neutral gender. They can be interpreted as automata, institutions or so on. Therefore we will avoid choosing their gender every time.
} 
by the equilibrium payoffs, with just the symmetric payoff being excluded.

There is a crucial step in the design of the negotiation model that is responsible for this asymmetry, and we can enunciate it by using the same authors' words:

"The key modeling issue is the specification of what happens if there is no agreement and, as a consequence, the game moves to the next stage. It is at this point that subgroups are made to matter by allowing for the possibility of partial breakdown of negotiations. Clearly, there are many ways to model such a partial breakdown. In the body of this paper we concentrate on a particular and simple class: disagreement puts only the proposer in jeopardy. That is, after his proposal is rejected, the proposer may cease to be an active participant."

Note that the cause of a rejection is due to the fact that the proposer offers less than what the responder expect to obtain. But who is the player responsible for such a breakdown? The proposer, offering too little, or the responder rejecting the offer because he wish for too much? An anonymous rule should not specify who is to blame for this breakdown, except if the rule itself computes what the right offer must be; but in that case the rule determines directly the right outcome without the help of the players. The HM-model identifies the proposer as being the only player responsible for the lack of agreement, giving him a chance $(1-\rho)$ to live the game after a rejected proposal.

In this paper we show that it is possible to yield strategic support for single-valued NTU-solutions, by changing the breakdown procedure, in such a way that, after rejection, the probability of leaving the game will be the same for all players (proposer and responders), making all of them responsible for the lack of agreement. There are several ways in which this can be done. We show two of them, which differ from the HM-model only in the rules of breakdown.

The simplest way to make this type of modification was already mentioned in Hart and 
Mas-Colell (1996a), Section 6. There, they propose several modifications of the bargaining procedure, and this is the case (d) from their list:

- Random removal. All players (proposer and responders) drop out with equal probability. The player that leaves the game receives a payoff of zero, and the rest restart the bargaining process.

The authors mention that in the TU-case "The resulting solution is different from the previous ones (thus, it is neither the Shapley value nor the equal split solution ${ }^{6}$ ). However, for a large $n$, it is easy to see that it is close to the equal split solution".

Nevertheless, the interest of this modification relapses into the solution obtained in the NTU-case. It satisfies both requirements mentioned above: Single-valuedness and symmetry. Moreover, it yields the Nash bargaining solution in pure bargaining games, and the solidarity value of Nowak and Radzik (1994) in TU-games.

The second modification proposed is a bit more elaborate, but has the advantage that the solution obtained fits into the Shapley value generalization program.

- Random marginal. A new proposer is chosen (among all of them) with equal probability. The proposer makes an ultimatum offer. If the rest of the players agree, this offer is the final payoff. If any player rejects, the proposer drops out of the game (receiving zero) and we restart the bargaining process with the remaining players.

Thus, in the ultimatum offer, the proposer knows for sure that they will leave the game in case of rejection. The difference between the random removal and random dictator is that, in random removal the player selected leaves the game directly, and in the random dictator, the player selected makes an offer, and it is only under rejection that the player leaves the game.

Now, the resulting solution supported by this bargaining procedure yields a new so-

\footnotetext{
${ }^{6}$ That is, the payoffs are shared equally beween the players of the coalition.
} 
lution in the NTU-case that it is again single-valued, and satisfies symmetry. When we deal with TU-games the Shapley value is selected, and in Hyperplane games it is the Consistent NTU-value. The price to pay relies on the fact that in pure bargaining problems the solution obtained is different from the Nash bargaining solution. The point selected is the maximization of utility gains from a breakdown point, so it is similar to the Nash solution (maximization of utility gains from the disagreement point), and the breakdown point is an average of the ideal points, from which the Kalai-Smorodinsky (1975) solution is based. So it has elements of both solutions in its definition.

Following this Introduction, Section 2 is devoted to preliminary definitions and notations. Section 3 presents the bargaining model of Hart and Mas-Colell and the modifications that yield the random marginal and the random removal bargaining models. The proposals corresponding to an equilibrum, for both bargaining models, are characterized in NTU-games. In Section 4 some two-person games are used to see the similarities and differences of the limit points associated to these models. Sections 5 and 6 are devoted to the characterization of the random marginal and random removal values in TU-games an NTU-games respectively. Finally, Section 7 explores in large games the connections between the random marginal value and the Shapley NTU-value, and between the random removal value and the equal split value.

\section{Definitions}

Let $N=\{1, \ldots, n\}$ be a finite set of players. A coalition is a subset of $N$. let $P(N)$ be the set of all coalitions of $N$. The cardinality of a coalition $S$ is denoted by $s$. If $x, y \in \mathbb{R}^{N}$, we write $x \geq y$ if $x_{i} \geq y_{i}$ for all $i \in N$, and $x>y$ if $x_{i}>y_{i}$ for all $i \in N$. Given two

vectors $x, y \in \mathbb{R}^{N}$, we use the notation $x \cdot y:=\sum_{i \in N} x_{i} y_{i}$, and $x * y:=\left(x_{i} y_{i}\right)_{i \in N}$. If 
$x \in \mathbb{R}^{N}$ and $\emptyset \neq S \subset N$, we write $x^{S}$ as the restriction of $x$ to $S$, i.e., $x^{S}=\left(x_{i}\right)_{i \in S} \in \mathbb{R}^{S}$. Let $\mathbb{R}_{+}^{N}:=\left\{x \in \mathbb{R}^{N} \mid x \geq 0\right\}$ and $\mathbb{R}_{++}^{N}:=\left\{x \in \mathbb{R}^{N} \mid x>0\right\}$. Let $A \subset \mathbb{R}^{N}, A$ is called comprehensive if $A-\mathbb{R}_{+}^{N} \subset A$. The boundary of $A$ is denoted by $\partial A$. We say that the boundary is non level if for all $x \in \partial A$ it holds that $\{x\}-\mathbb{R}_{+}^{N} \cap \partial A=\{x\}$.

A non-transferable utility game (NTU-game for short), is a map $V$ assigning to each coalition $S, \emptyset \neq S \subset N$, a subset $V(S) \subset \mathbb{R}^{S}$ of attainable payoff vectors for players in $S$. several regularity conditions are imposed such as:

(A.1) $V(S)$ is non-empty, closed, convex and comprehensive.

(A.2) $\partial V(S)$ is non level.

(A.3) $0 \in V(S)$ and $V_{0}(S):=V(S) \cap \mathbb{R}_{+}^{S}$ is bounded.

(A.4) Monotonicity: $V_{0}(S) \times\left\{0^{T \backslash S}\right\} \subset V_{0}(T)$ whenever $S \subset T$.

(A.5) Positively smoothness: For each $S \subset N$, at each $x$ of $\partial V(S)$ there exists a unique supporting hyperplane to $V(S)$ (i.e. $V(S) \subset\left\{y \in \mathbb{R}^{S}: \lambda \cdot y \leq \lambda \cdot x\right\}$ ) such that $\lambda \in \mathbb{R}_{++}^{S}$.

The assumption A.4 is just the extension on NTU-games of the classical Monotonicity assumption for TU-games. The class of all games that satisfy A.1, A.2, A.3, and A.4, is denoted by $\mathcal{G}$.

For each $i \in N$, let $r^{i}:=\max \{x: x \in V(i)\}$, and let $r=\left(r^{i}\right)_{i \in N} \in \mathbb{R}^{N}$. Some relevant classes of NTU-games are:

1. Transferable utility games (TU-games), when for each coalition $S$ there is a number $v(S)$ such that $V(S)=\left\{x \in \mathbb{R}^{S}: \sum_{i \in S} x^{i} \leq v(S)\right\}$ for all $S \subset N$. Risk neutral players who use a totally divisible good to make the coalitional payoffs is an example of this type of games. If $V$ is a TU-game, it will be denoted also by $v$.

2. Hyperplane games (H-games), when $\partial V(S)$ is a hyperplane for all $S \subset N$. That is, for each coalition $S$ there exists a number $v(S)$ and a vector $\lambda_{S} \in \mathbb{R}_{++}^{S}$ such that $V(S)=\left\{y \in \mathbb{R}^{S}: \lambda_{S} \cdot y \leq v(S)\right\}$. For example, prize games can be modeled in this way: 
Each coalition $S \subset N$ has a prize $\pi_{S}$. The prize $\pi_{S}$ is indivisible, and only one member of $S$ can receive it. The feasible set of each coalition $S$ consists of all lotteries over which players in $S$ get the prize $\pi_{S}$ (for more details see Hart, 1994).

3. Pure Bargaining games (PB-games), when $0 \in \partial V(S)$, for all $S \neq N$. Pure Bargaining games are usually described by a pair $(0, V(N))$, where $V(N)$ is the utility feasible set attainable by unanimity agreements of all members of $N$, and 0 is the utility feasible payoffs vector obtained in case of disagreement. The fact that no other than the grand coalition can make agreements is precisely reflected by $0 \in \partial V(S)$, for all $S \neq N$.

Remark. In the normalization assumption A.3, it is worth noting that we have been making the implicit assumption that the utilities are previously normalized in such a way that when any player leaves the game, the payoff that it obtains is zero. The payoff $r^{i}$ is what player $i$ obtains in the game if the remaining $N \backslash i$ players have left the game. Hence a two-person NTU-game will be a pure bargaining game only when $r=0$.

A payoff configuration is a family $\mathbf{x}=\left(x_{S}\right)_{S \subset N}$ where $x_{S} \in \mathbb{R}^{S}$ for all $S \subset N$. A value on $\mathcal{G}$ is a function $\phi$ that assigns a unique payoff configuration to each game belonging to $\mathcal{G}$. Given $V \in \mathcal{G}$, and $S \subset N$, the vector $\phi_{S}(V)$ is called the value of $V$ for $S$. A solution on $\mathcal{G}$ is a mapping $\Psi$ that assigns a set of payoff configurations to each game belonging to $\mathcal{G}$. For notational simplicity, we use $S \backslash i$ and $S \cup i$ instead of $S \backslash\{i\}$ and $S \cup\{i\}$ respectively.

\section{The bargaining model}

We describe here the multilateral bargaining procedures, based on the random removal and random marginal approach, within the general setting of NTU-games. These two approaches are based on the HM-model, and hence have a similar structure. For this 
reason, in order to see more clearly the differences and similarities between them, we describe the three models at the same time.

We specify now the sequential noncooperative games which specify the rules of bargaining. The three models only differ in what happens if breakdown occurs ${ }^{7}$.

Let an NTU-game $V \in \mathcal{G}$ and $0 \leq \rho<1$ be a fixed parameter:

In each round there is a set $S \subset N$ of active players, and a proposer $i \in S$. In the first round, the active set is $S=N$. The proposer is chosen at random out of $S$, with all players in $S$ being equally likely to be selected. The proposer makes a feasible offer $a_{S, i} \in V(S)$. If all members of $S$ accept it -they are asked in some prespecified order- then the game ends with these payoffs. If it is rejected by even one member of $S$, then, with probability $\rho$, we move to a next round where the set of active players is again $S$ and, with probability $1-\rho$, breakdown occurs.

HM-breakdown: The proposer $i$ drops out -leaves the game, receiving a payoff of zero- and we move to a next round where the set of active players becomes $S \backslash i^{8}$.

$R R$-breakdown (random removal): A player $j$ is chosen at random from $S$ to drop out, being equally likely to be selected, and we move to a next round where the set of active players becomes $S \backslash j$.

$R M$-breakdown (random marginal): A player $j$ is chosen at random from $S$ to make an ultimatum offer, being equally likely to be selected: Proposer $j$

\footnotetext{
${ }^{7}$ We call HM-model of bargaining ( RR-model, RM-model, respec.) when the breakdown follows the HM-breakdown (RR-breakdown, RM-breakdown, respec.) rule.

${ }^{8}$ In what follows we write $S \backslash i$ and $S \cup i$ for $S \backslash\{i\}$ and $S \cup\{i\}$ respectively.
} 
makes a feasible offer $u_{S, j} \in V(S)$. If all members of $S$ accept it -they are asked in some prespecified order- then the game ends with these payoffs. If it is rejected by even one member of $S$, then $j$ drops out and we move to a next round where the set of active players becomes $S \backslash j$.

The negotiation games have potentially infinitely many periods, and with more than two active players, it is well known that many subgame perfect equilibria strategies appear. Hence, as usual, we restrict ourselves to considering only stationary strategies. Therefore, the choice at each stage only depends on the set of active players $S$ and on the current proposer $i$. Given a profile of stationary strategies, denoted by $a_{S, i}(\rho)$, for $i \in S \subset N$, the proposal when the set of active players is $S$ and the proposer is $i$. The average of these proposals is defined by $a_{S}(\rho):=(1 / s) \sum_{i \in S} a_{S, i}(\rho)^{9}$.

We recall first the equations that characterize the stationary subgame perfect equilibrium (SP) of the HM-model (Proposition 1, Hart and Mas-Colell, 1996a).

Proposition 1 The proposals corresponding to an SP equilibrium are always accepted, and they are characterized by:

(HM.1) $\quad a_{S, i}(\rho) \in \partial V(S)$ for all $i \in S \subset N$, (HM.2) $\quad a_{S, i}^{j}(\rho)=\rho a_{S}^{j}(\rho)+(1-\rho) a_{S \backslash i}^{j}(\rho)$ for all $i, j \in S \subset N$ with $i \neq j$.

Moreover, these proposals are nonnegative.

The proposition says that $i$ makes offers such that they will obtain their maximum payoff compatible by giving to the rest of players what they would expect to obtain in the continuation of the game if the offer were rejected, i.e., for every responder $j \neq i$, they will get their expected payoff $a_{S}^{j}(\rho)$ when the active player set is $S$ again, with probability

\footnotetext{
${ }^{9}$ We denote the cardinality of a finite set $S$ by $s$.
} 
$\rho$, and $a_{S \backslash i}^{j}(\rho)$ when the active player set is $S \backslash i$, with probability $(1-\rho)$ of $i$ dropping out.

In the next two propositions we characterize the conditions of an SP equilibrium in the RR and RM-breakdown models. They only differ in what players can expect to get in the continuation of the game in case of breakdown.

For any $S \subset N$, define

$$
d_{S}^{j}(\rho):=\frac{1}{s} \sum_{k \in S \backslash j} a_{S \backslash k}^{j}(\rho), \quad j \in S .
$$

Therefore $d_{S}(\rho)=(1 / s) \sum_{j \in S}\left(0, a_{S \backslash j}(\rho)\right)$, where $\left(0, a_{S \backslash j}(\rho)\right) \in \mathbb{R}^{S}$ is the payoff vector in which player $j$ drops out receiving 0 , and players $k \neq j$ receive $a_{S \backslash j}^{k}(\rho)$.

Proposition 2 The proposals corresponding to an SP equilibrium in the RR-breakdown model are always accepted, and are characterized by:

(RR.1) $\quad a_{S, i}(\rho) \in \partial V(S)$ for all $i \in S \subset N$,

(RR.2) $\quad a_{S, i}^{j}(\rho)=\rho a_{S}^{j}(\rho)+(1-\rho) d_{S}^{j}(\rho)$ for all $i, j \in S \subset N$ with $i \neq j$.

Moreover, these proposals are nonnegative.

For the RM-breakdown model the proposition is very similar. For any $S \subset N$, we define $u_{S}(\rho):=(1 / s) \sum_{j \in S} u_{S, j}(\rho)$, where $u_{S, j}(\rho) \in \mathbb{R}^{S}$ is the ultimatum offer when player $j \in S$ is selected as proposer in the RM-breakdown.

Proposition 3 The proposals corresponding to an SP equilibrium in the RM-breakdown model are always accepted, and are characterized by:

(RM.1) $\quad a_{S, i}(\rho) \in \partial V(S)$ for all $i \in S \subset N$,

(RM.2) $\quad a_{S, i}^{j}(\rho)=\rho a_{S}^{j}(\rho)+(1-\rho) u_{S}^{j}(\rho)$ for all $i, j \in S \subset N$ with $i \neq j$,

(RM.3) $\quad u_{S, i}(\rho) \in \partial V(S)$ for all $i \in S \subset N$, 
(RM.4) $\quad u_{S, i}^{j}(\rho)=a_{S \backslash i}^{j}(\rho)$ for all $i, j \in S \subset N$ with $i \neq j$.

Moreover, $a_{S}(\rho) \geq u_{S}(\rho)$, and $a_{S, i}^{i}(\rho) \geq u_{S}^{i}(\rho) \geq 0$, for all $i \in S \subset N$.

The proof of Propositions (RR) and (RM) are rather similar of the HM-model (see Proposition 1, in Hart and Mas-Colell, 1996a). In particular, Proposition (RR) is just case (d) of proposition 9, of Hart and Mas-Colell (1996a). So we will pay attention only to the RM-model.

Proof. It is proceeded by induction. It can be easily checked for the 1-player case. Let $\left(a_{S, i}(\rho), u_{S, i}(\rho)\right)$, for $i \in S \subset N$, be the proposals of a given SP equilibrium. Assume by induction hypothesis that RM.1-RM.4 are satisfied for $S \neq N$. We see firstly that, in case of breakdown, $u_{S, i}(\rho)$ satisfies RM.3 and RM.4 for any $i \in N$. Because it is assumed that $a_{S, i}(\rho) \in \partial V(S)$ and $a_{S, i}(\rho) \geq 0$ for $i \in S \neq N$, monotonicity and convexity imply that $a_{S}(\rho) \in V_{0}(S)$ for all $S \neq N$. Let $i \in N$ be the proposer of an ultimatum offer in case of breakdown. Because players $j$ in $N \backslash i$ can guarantee $a_{N \backslash i}^{j}(\rho)$ by rejection, they only accept offers such that $u_{N, i}^{j}(\rho) \geq a_{N \backslash i}^{j}(\rho)$. Hence the best player $i$ can do is to offer $u_{N, i}^{j}(\rho)=a_{N \backslash i}^{j}(\rho)$ for all $j \in N \backslash i$. Let $u_{N, i}^{i}(\rho)$ be such that $u_{N, i}(\rho) \in \partial V(N)$. by Monotonicity it holds that $u_{N, i}^{i}(\rho) \geq 0$. if $u_{N, i}^{i}(\rho)>0$, the best player $i$ can do is to offer $u_{N, i}(\rho)$, which will be accepted by all $j \in N \backslash i$. If $u_{N, i}^{i}(\rho)=0$, player $i$ is indifferent between offering $u_{N, i}(\rho)$, which will be accepted, and offering a different proposal $b_{N, i}(\rho) \neq u_{N, i}(\rho)$ such that $b_{N, i}^{i}(\rho)>0$. In this latter case, some player $j \neq i$ must necessarily have $b_{N, i}^{j}(\rho)<a_{N \backslash i}^{j}(\rho)$, because A.1 and A.2 imply that $\partial V(N)$ coincides with the Pareto frontier of $V(N)$, therefore $b_{N, i}(\rho)$ will be rejected by player $j$. In both cases $i$ will obtain 0 and the rest of the players $j \in S \backslash i$ obtain $a_{N \backslash i}^{j}(\rho)$, which again coincides with $u_{N, i}(\rho)^{10}$. Let $u_{N}(\rho):=(1 / n) \sum_{i \in N} u_{N, i}(\rho)$, by construction $u_{N, i}(\rho) \in \partial V_{0}(N)$ for all $i \in N$, then $u_{N}(\rho) \in V_{0}(N)$ by convexity.

\footnotetext{
${ }^{10}$ In this indifferent case, mixed strategies also yield the same outcome.
} 
Denote by $c_{N}$ the expected payoff vector for the members of $N$. By convexity it must be $c_{N} \in V(N)$, and also $\rho c_{N}(\rho)+(1-\rho) u_{N}(\rho) \in V(N)$. Define the vector $d_{N, i}(\rho)$ such that $d_{N, i}(\rho) \in \partial V(N)$ and $d_{N, i}^{j}(\rho)=\rho c_{N}^{j}(\rho)+(1-\rho) u_{N}^{j}(\rho)$ for all $j \in N \backslash i$. Thus,

$d_{N, i}^{i}(\rho) \geq \rho c_{N}^{i}(\rho)+(1-\rho) u_{N}^{i}(\rho)$. For $j \neq i, d_{N, i}^{j}(\rho)$ is the expected payoff of $j$ following a rejection of $i$ 's proposal. therefore $d_{N, i}(\rho)$ is the best proposal that will be accepted if $i$ is the proposer. Moreover, any proposal of $i$ which is rejected yields to $i$ at most $\rho c_{N}^{i}(\rho)+(1-\rho) u_{N}^{i}(\rho) \leq d_{N, i}^{i}(\rho)$. Hence, player $i$ will propose $a_{N, i}(\rho)=d_{N, i}(\rho)$ and the proposal will be accepted, and therefore $c_{N}(\rho)=a_{N}(\rho)$. To see that $a_{N}(\rho) \geq u_{N}(\rho)$ note that the following strategy will guarantee to any $i$ a payoff of at least $u_{N}^{i}(\rho)$ : accept only if offered at least $u_{N}^{i}(\rho)$, and, when proposing, propose $u_{N}(\rho)$. This implies that $a_{N}^{i}(\rho) \geq u_{N}^{i}(\rho)$, and then $a_{N, i}^{i}(\rho) \geq \rho a_{N}^{i}(\rho)+(1-\rho) u_{N}^{i}(\rho) \geq u_{N}^{i}(\rho)$. Given the strategies of the other players, $i$ can not increase their payoff from proposals that are accepted, and making proposals that were systematically rejected they could only yield the chance to go to the breakdown stage, which gives $u_{N}(\rho)$ as expected payoffs. Whereas the suggested strategies yields $a_{N}(\rho)$ which is a better outcome $\left(a_{N}(\rho) \geq u_{N}(\rho)\right)$.

\section{A two-person example}

In the first example we illustrate the problem in which players can be involved when they follow a solution concept that does not satisfy the uniqueness requirement.

Let us suppose there are two players who both claim an indivisible good. In addition, assume that this good can be owned either by only one player or shared by both simultaneously. The players have the same preferences and they are risk neutral. The set of pure feasible outcomes is $A=\left\{O_{1}, O_{2}, S, E\right\}$, where $O_{i}(i=1,2)$ means that the good is only for player $i, S$ means that the good is shared by both players, and $E$ means that the 
good is for nobody. We normalize the utilities as follows:

$$
\begin{aligned}
u^{1}\left(O_{1}\right) & =u^{2}\left(O_{2}\right)=75, \\
u^{1}(S) & =u^{2}(S)=50, \\
u^{1}(E) & =u^{2}(E)=u^{1}\left(O_{2}\right)=u^{2}\left(O_{1}\right)=0 .
\end{aligned}
$$

Here the set of players is $N=\{1,2\}$ and the characteristic function $V$ is built as follows: If a player renounces their claim, they "leave" the game, and then the good is for the other, hence

$$
V(\{i\})=\left\{x: x \leq r^{i}\right\} \text { where } r^{i}=75, i \in N
$$

When both players claim for the good, they can either agree on any pure outcome in $A$, or on any lottery in $A$ (for example, tossing a coin to decide if the good is only for player $i$ or $\left.j:\left[O_{1}, p_{1}=\frac{1}{2} ; O_{2}, p_{2}=\frac{1}{2}\right]\right)$. Therefore the feasible expected payoffs that both claimants can guarantee by cooperation are established by the convex hull of $u(A)=$ $\{(50,50),(75,0),(0,75),(0,0)\}$. Therefore,

$$
V(N)=\operatorname{conv}(u(A))-\mathbb{R}_{+}^{2},
$$

("conv" denotes "convex hull"). The sets $V(\cdot)$ are also comprehensive (utility is freely disposable). $V(N)$ is represented in Figure 1. 


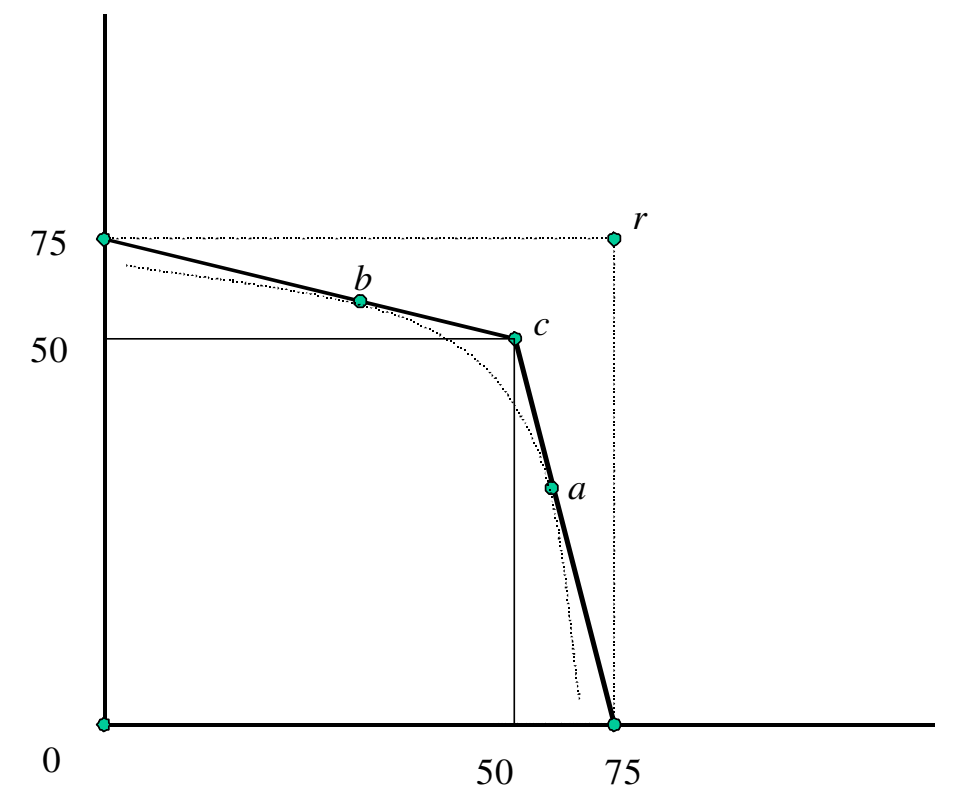

Three solution points.

Now applying the Consistent NTU-solution to this example, the solution selects three possible outcomes: $a=(56.25,37.5), b=(37.5,56.25)$, and $c=(50,50)^{11}$.

Apply now the Hart and Mas-Colell (1996a) bargaining procedure to our example. The equilibrium equations which determine the proposals in $N$ are $x_{N, i}(\rho) \in \partial V(N)$, for all $i \in N$, and $x_{N, i}^{j}(\rho)=\rho x_{N}^{j}(\rho)+(1-\rho) r^{j}$, for $j \neq i$, where $x_{N}(\rho)$ is the expected vector payoffs for coalition $N$, i.e., $x_{N}(\rho)=\frac{1}{2} x_{N, 1}(\rho)+\frac{1}{2} x_{N, 2}(\rho)$.

That is, player $i$ offers to player $j$ just what they will get in the case of rejecting the proposal: $x_{N}(\rho)$ in case the game repeats, and $r^{j}$ in case breakdown happens, being player $i$ the only claimant that remains in the game.

\footnotetext{
${ }^{11}$ It can easily be checked that these three points also belong to the set of Shapley and Harsanyi NTU-solutions of this game.
} 
It can be checked that equations both equations imply that

$$
\left(x_{N, 1}^{1}(\rho)-r^{1}\right)\left(x_{N, 1}^{2}(\rho)-r^{2}\right)=\left(x_{N, 2}^{1}(\rho)-r^{1}\right)\left(x_{N, 2}^{2}(\rho)-r^{2}\right) \text {, }
$$

which, in our example, yield two different solutions: $\left\{a_{N, 1}(\rho), a_{N, 2}(\rho)\right\}$ and $\left\{b_{N, 1}(\rho), b_{N, 2}(\rho)\right\}$ that, when $\rho \rightarrow 1$, they converge to $a$ and $b$ respectively (see Figure 2).

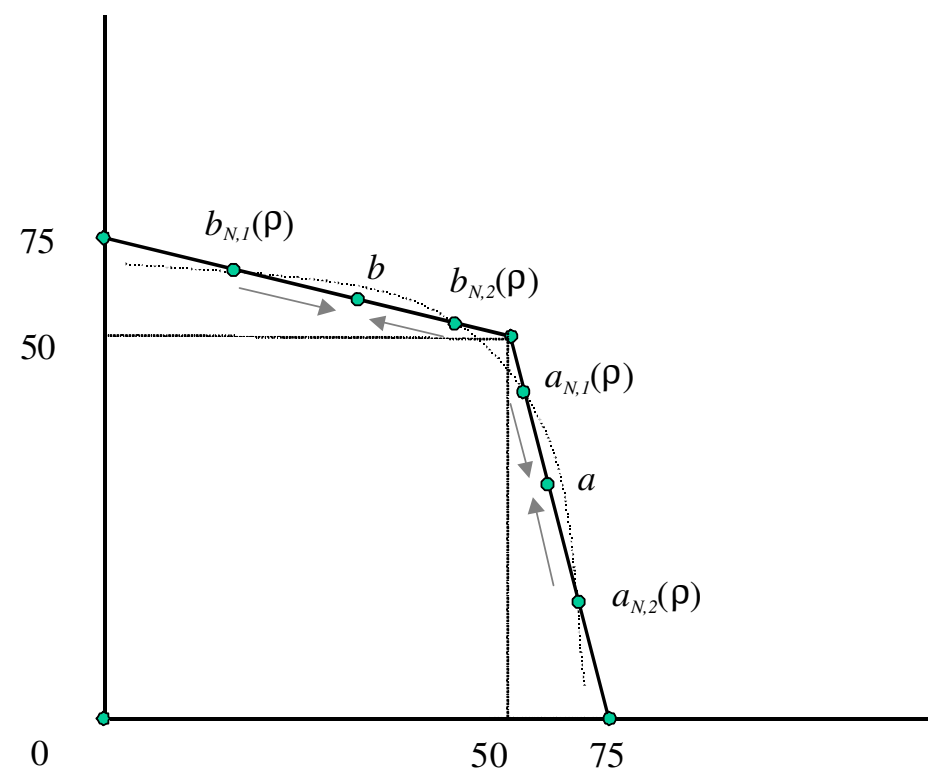

Limit points when $\rho \rightarrow 1$.

First, note that this bargaining procedure does not always allow an approximation to all payoff solutions: In our example, point $c=(50,50)$ is excluded. Secondly, we have multiplicity: we can approximate either $a$ or $b$. If we have no previous reasons to discriminate between claimants 1 and 2, a way to solve this impasse is to choose with a fair lottery between $a$ and $b$ by tossing a coin. But therefore, the expected payoffs are $(46.875,46.875)$ that are Pareto dominated by $(50,50)$.

Consider now the two alternative breakdown rules proposed. 
Random removal $(R R)$ : Both players have the same probability of living the game. If player $i$ leaves the game, their payoff is zero, and player $j$ receives their claim $r^{j}$. Therefore the expected payoffs vector $d$ is

$$
d=\frac{1}{2}\left(0, r^{2}\right)+\frac{1}{2}\left(r^{1}, 0\right)=\frac{1}{2}(0,75)+\frac{1}{2}(75,0)=(37.5,37.5) .
$$

Random marginal (RM): Both players have the same probability of being the proposer of an ultimatum payoff vector. If player $i$ proposes $u_{i} \in V(N)$, player $j$ is asked if they agree or dissent. If agrees, $u_{i}$ is the final payoff. If dissents, player $i$ leaves the game, receiving a payoff of zero, and player $j$ receives their claim $r^{j}$. The equilibrium proposals $u_{i}$ are characterized by $u_{i} \in \partial V(N)$, for all $i \in N$, and $u_{i}^{j}=r^{j}$, for $j \neq i$. Therefore, it follows that, the expected payoffs vector $u$ is $u=\frac{1}{2} u_{1}+\frac{1}{2} u_{2}$. In our example, if a proposer $i$ is compelled to make an ultimatum offer to $j$, they must offer $r^{j}=75$ units, because this is what the other claimant would obtain if the proposer is forced to leave the game in case of rejection. Hence the expectations are

$$
u=\frac{1}{2}(0,75)+\frac{1}{2}(75,0)=(37.5,37.5) .
$$

Therefore, in this particular example, the breakdown expected payoffs $d$ and $u$ coincide, and we denote this point $(37.5,37.5)$ by $h$. It follows that in both bargaining models, the equilibrium equations which determine the proposals in $N$ are $x_{i}(\rho) \in \partial V(N)$, for all $i \in N$, and $x_{i}^{j}(\rho)=\rho x_{N}^{j}(\rho)+(1-\rho) h^{j}$, for $j \neq i$, where $x_{N}(\rho)=\frac{1}{2} x_{N, 1}(\rho)+\frac{1}{2} x_{N, 2}(\rho)$. It can be easily checked that

$\left(x_{N, 1}^{1}(\rho)-h^{1}\right)\left(x_{N, 1}^{2}(\rho)-h^{2}\right)=\left(x_{N, 2}^{1}(\rho)-h^{1}\right)\left(x_{N, 2}^{2}(\rho)-h^{2}\right)=(2-\rho) \rho\left(x_{N}^{1}(\rho)-h^{1}\right)\left(x_{N}^{2}(\rho)-h^{2}\right)$.

Therefore, when $\rho \rightarrow 1$, we have $c_{i}(\rho) \rightarrow c(\rho)$ for $i=1,2$, and $c(\rho) \rightarrow c=(50,50)$ Hence, we fall into the classical approach to the Nash Bargaining solution from the disagreement 
point $h$, which, in our example, is the point that maximizes the product of the utility gains from the reference point $h$ (see Figure 3).

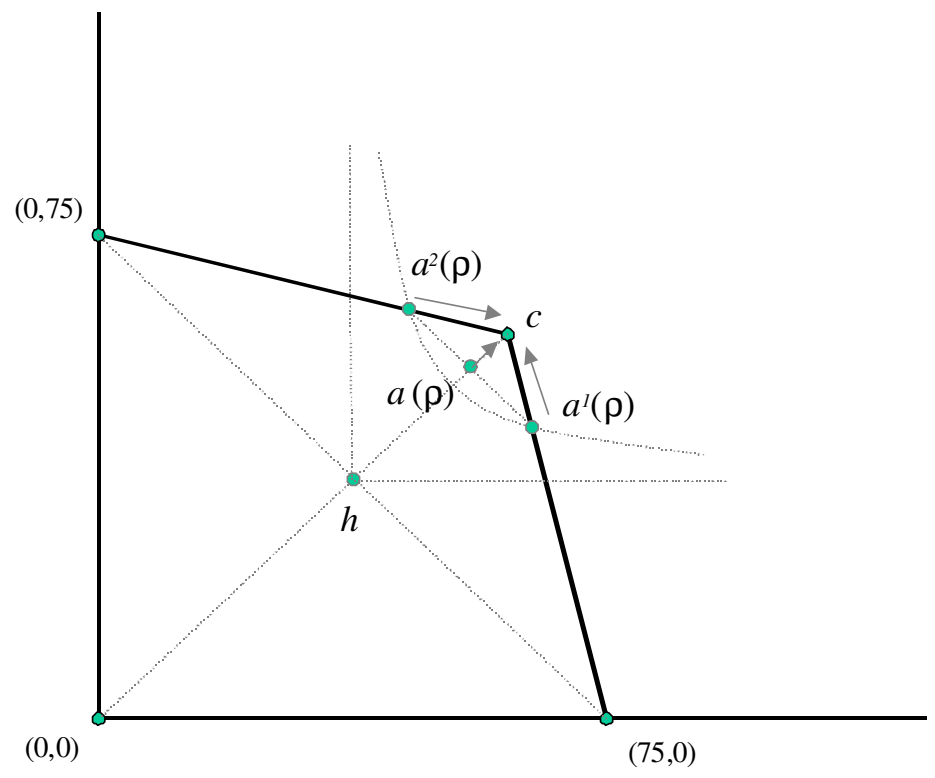

Limit point in the random marginal and random removal models.

Although in this example our procedure yields, in the limit, a point that belongs to the set of Consistent NTU-value allocations, i.e. the point $c=(50,50)$, this fact is not true in general, as can be seen in the following two examples.

In the pure bargaining game of Figure 4, the random marginal model yields at the limit the point $\zeta=(60,40)$ that maximizes the product of utility gains taken from the breakdown point $u=(50,30)$. The random removal model yields in the limit the Nash solution (which coincides with the Shapley, Harsanyi and Consistent NTU-value), i.e., point $N=(50,50)$ that maximizes the product of utility gains from disagreement point $d=(0,0)$. 


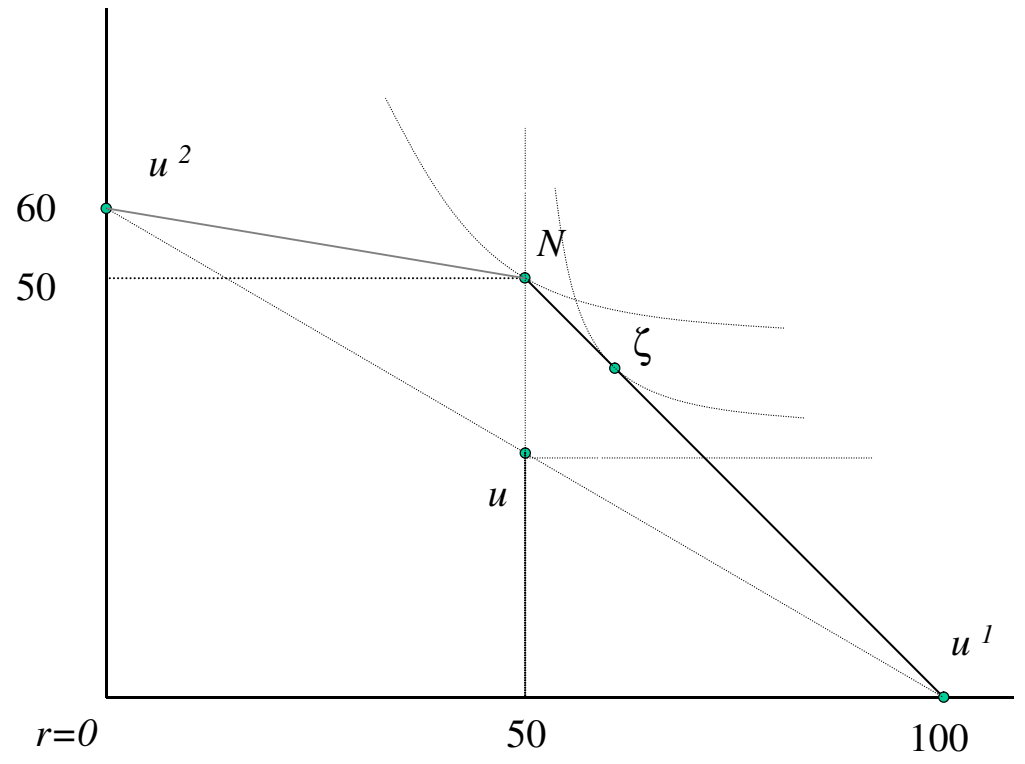

Differences between the random marginal and the random removal payoffs.

The following two-person TU-game is an additive game, i.e., $v(N)=r^{1}+r^{2}$. The ultimatum offers of the breakdown random marginal are $u_{N, 1}=u_{N, 2}=\left(r^{1}, r^{2}\right)=u$, and because $u \in \partial V(N)$ it holds that $\zeta=u$. Within the random removal model, the expected payoffs vector in case of breakdown is

$$
d=\frac{1}{2}\left(0, r^{2}\right)+\frac{1}{2}\left(r^{1}, 0\right)=\frac{1}{2} r
$$

which yields, in the limit, the bargaining payoffs

$$
\psi^{i}=\frac{1}{2} r^{i}+\frac{1}{2}\left(\left(r^{i}+r^{j}\right)-\frac{1}{2} r^{i}-\frac{1}{2} r^{j}\right)=\frac{3}{4} r^{i}+\frac{1}{4} r^{j}, \quad i, j \in N=\{1,2\} .
$$

This shows that the solution associated to the random removal model does not satisfy Additivity in TU-games. 


\section{TU-games}

In this section we analyze the behavior of the RM and RR breakdown models in the case of transferable utility games. The results of this section are particular cases of the results obtained in the next section for NTU-games. Here we find the main difference of both models: The RM-model supports the Shapley value, and the RR-model supports the solidarity value.

Random marginal

Let $v$ be a TU-game. For each coalition $S \subset N$ and player $i \in S$, let

$$
\partial^{i}(v, S):=v(S)-v(S \backslash i)
$$

be the marginal contribution of player $i$ to coalition $S$ in the TU-game $v$. The Shapley value in the game $v$ is the payoff configuration $\varphi=\left(\varphi_{S}(v)\right)_{s \subset N}$ defined by

$$
\varphi_{S}^{i}(v)=\sum_{\substack{T \subset S \\ T \ni i}} \frac{(s-t) !(t-1) !}{s !} \partial^{i}(v, T), \quad(i \in S \subset N) .
$$

Alternatively, this value can be obtained recursively ${ }^{12}$ by

$$
\varphi_{S}^{i}(v)=\frac{1}{s} \partial^{i}(v, S)+\sum_{j \in S \backslash i} \frac{1}{s} \varphi_{S \backslash j}^{i}(v), \quad(i \in S \subset N),
$$

starting with

$$
\varphi_{\{i\}}^{i}(v)=r^{i}, \text { for all } i \in N
$$

In the Proposition (RM) we have seen that the rules of the bargaining guarantee that the equilibrium payoffs $a^{S}$ of the negotiation stages will always be greater than or equal to the breakdown payoffs $u^{S}$, for all $S \subset N$. This fact implies the next straightforward Theorem.

\footnotetext{
${ }^{12}$ See in Hart and Mas-Colell (1996a), the Remark of Section $4 .$.
} 
Theorem 4 Let $\left(a_{S}(\rho), u_{S}(\rho)\right)_{S \subset N}$ be the equilibrium payoffs configuration associated to the RM-breakdown model. Then $\mathbf{a}(\boldsymbol{\rho})=\left(a_{S}(\rho)\right)_{S \subset N}$ coincides with the Shapley value for TU-games, for any $\rho$.

Proof. Let $V$ be a TU-game. By (RM.3) $\sum_{j \in S} u_{S, i}^{j}(\rho)=v(S)$, for all $i \in S$, hence $\sum_{j \in S} u_{S}^{j}(\rho)=v(S)$, for all $S \subset N$. Moreover, $a_{S, i}(\rho) \geq u_{S}(\rho)$, and $\sum_{j \in S} a_{S, i}^{j}(\rho)=v(S)$, for all $i \in S$; then $a_{S, i}(\rho)=a_{S}(\rho)=u_{S}(\rho)$ and $\sum_{j \in S} a_{S}^{j}(\rho)=v(S)$. Therefore

$$
a_{S}^{i}(\rho)=\frac{1}{s} u_{S, i}^{i}(\rho)+\sum_{j \in S \backslash i} \frac{1}{s} a_{S \backslash j}^{i}(\rho),(i \in S) .
$$

By (RM.3), (RM.4), and $\sum_{j \in S \backslash i} a_{S \backslash i}^{j}(\rho)=v(S \backslash i)$, we have that

$$
u_{S, i}^{i}(\rho)=\partial^{i}(v, S)
$$

The payoffs of the single coalitions $\{i\}$, are $a_{\{i\}, i}^{i}(\rho)=r^{i}$, for all $i \in N$. Thus they are independent of $\rho$. This implies that the ultimatum payoffs for two-player coalitions are also independent of $\rho$, and equal to

$$
a_{S}^{i}=\frac{1}{2} \partial^{i}(v, S)+\frac{1}{2} r^{i}, \quad(i \in S=\{i, j\}) .
$$

By increasing the size of the coalitions, this recursive argument shows that the equilibrium payoffs are independent of $\rho$, and equal to

$$
a_{S}^{i}=\frac{1}{s} \partial^{i}(v, S)+\sum_{j \in S \backslash i} \frac{1}{s} a_{S \backslash j}^{i}, \quad(i \in S \subset N) .
$$

Therefore $a_{S}^{i}=\varphi_{S}^{i}(v)$ for all $i \in S \subset N$.

Remark. It is interesting to note the differences with the results for the HM-model in the TU-case. In the HM-model, we have that $a_{S}(\rho)=\varphi_{S}(v)$, and $a_{S, i}(\rho) \rightarrow \varphi_{S}(v)$ when $\rho \rightarrow 1$, for all $i \in S \subset N$. It means that in the RM-model there is no difference between 
being the proposer or the responder, whereas in the HM-model this is not the case. So, in the TU-case, in the RM-model the bargaining part (in which players make proposals and counterproposals) is irrelevant, because they are strongly determined by the breakdown part. For this reason, if one wishes to support the Shapley value, the bargaining can be simplified with only two moves, for each round of active players set $S$ : First choose randomly a proposer out of $S$ (equally likely). If the proposal is rejected, then randomly choose again another proposer out of $S$ (equally likely) to make an ultimatum proposal. If it is rejected, then the proposer drops out of the game and proceed to a new round with the remaining players as the new active set.

\section{Random removal}

Let $v$ be a TU-game. For each coalition $S \subset N$, let

$$
\partial^{a v}(v, S):=\frac{1}{s} \sum_{i \in S} \partial^{i}(v, S)
$$

be the average marginal contribution of a player to coalition $S$ in the TU-game $v$. The solidarity value in the game $v$ is the payoff configuration $\psi=\left(\psi_{S}(v)\right)_{s \subset N}$ defined by

$$
\psi_{S}^{i}(v)=\sum_{\substack{T \subset S \\ T \ni i}} \frac{(s-t) !(t-1) !}{s !} \partial^{a v}(v, T), \quad(i \in S \subset N) .
$$

This value was introduced in Nowak and Radzik (1994). Similarly to the Shapley value, it can be easily checked that this value can be obtained recursively by

$$
\psi_{S}^{i}(v)=\frac{1}{s} \partial^{a v}(v, S)+\sum_{j \in S \backslash i} \frac{1}{S} \psi_{S \backslash j}^{i}(v), \quad(i \in S \subset N),
$$

starting with

$$
\psi_{\{i\}}^{i}(v)=r^{i}, \text { for all } i \in N
$$


Theorem 5 Let $\left(a_{S}(\rho)\right)_{S \subset N}$ be the equilibrium payoffs configuration associated to the RR-breakdown model. Then $\mathbf{a}(\boldsymbol{\rho})=\left(a_{S}(\rho)\right)_{S \subset N}$ coincides with the solidarity value for TU-games, for any $\rho$.

Proof. Let $V$ be a TU-game. By (RR.1), for any $i \in S \subset N$, we have

$$
a_{S, i}^{i}(\rho)=\left(v(S)-\sum_{j \in S \backslash i} a_{S, i}^{j}(\rho)\right),
$$

then

$$
a_{S}^{i}(\rho)=\frac{1}{s} a_{S, i}^{i}+\frac{1}{s} \sum_{j \in S \backslash i} a_{S, j}^{i}(\rho),
$$

which yields

$$
s a_{S}^{i}(\rho)=\left(v(S)-\sum_{j \in S \backslash i} a_{S, i}^{j}(\rho)\right)+\sum_{j \in S \backslash i} a_{S, j}^{i}(\rho) .
$$

Applying (RR.2),

$$
\begin{aligned}
s a_{S}^{i}(\rho)= & v(S)-\sum_{j \in S \backslash i}\left(\rho a_{S}^{j}(\rho)+(1-\rho) \frac{1}{s} \sum_{k \in S \backslash j} a_{S \backslash k}^{j}(\rho)\right)+\sum_{j \in S \backslash i}\left(\rho a_{S}^{i}(\rho)+(1-\rho) \frac{1}{s} \sum_{k \in S \backslash i} a_{S \backslash k}^{i}(\rho)\right) \\
= & v(S)-\sum_{j \in S \backslash i} \rho a_{S}^{j}(\rho)-\rho a_{S}^{i}(\rho)-\frac{1-\rho}{s} \sum_{j \in S \backslash i} \sum_{k \in S \backslash j} a_{S \backslash k}^{j}(\rho) \\
& +\sum_{j \in S \backslash i} \rho a_{S}^{i}(\rho)+\rho a_{S}^{i}(\rho)+\frac{1-\rho}{s} \sum_{j \in S \backslash i} \sum_{k \in S \backslash i} a_{S \backslash k}^{i}(\rho),
\end{aligned}
$$

which, applying (RR.1) again, yields

$$
s a_{S}^{i}(\rho)=v(S)-\frac{1}{s} \sum_{j \in S \backslash i} \sum_{k \in S \backslash j} a_{S \backslash k}^{j}(\rho)+\frac{s-1}{s} \sum_{k \in S \backslash i} a_{S \backslash k}^{i}(\rho) .
$$

Note that

$$
\sum_{j \in S \backslash i} \sum_{k \in S \backslash j} a_{S \backslash k}^{j}(\rho)=\sum_{k \in S \backslash i} a_{S \backslash i}^{k}(\rho)+\sum_{k \in S \backslash i} \sum_{j \in S \backslash i,} a_{S \backslash k}^{j}(\rho)=v(S \backslash i)+\sum_{k \in S \backslash i}\left(v(S \backslash k)-a_{S \backslash k}^{i}(\rho)\right) .
$$


Then we obtain

$$
s a_{S}^{i}(\rho)=v(S)-\frac{1}{s} \sum_{k \in S} v(S \backslash k)+\sum_{k \in S \backslash i} a_{S \backslash k}^{i}(\rho)=\frac{1}{s} \partial^{a v}(v, S)+\frac{1}{s} \sum_{k \in S \backslash i} a_{S \backslash k}^{i}(\rho) .
$$

The payoffs of the single coalitions $\{i\}$, are $a_{\{i\}, i}^{i}(\rho)=v(i)$, for all $i \in N$. So they are independent of $\rho$. A recursive argument shows that the average equilibrium payoffs $a_{S}^{i}$ are independent of $\rho$, and equal to

$$
a_{S}^{i}=\frac{1}{s} \partial^{a v}(v, S)+\sum_{k \in S \backslash i} \frac{1}{s} a_{S \backslash k}^{i},(i \in S \subset N) .
$$

Therefore $a_{S}^{i}=\psi_{S}^{i}(v)$ for all $i \in S \subset N$.

Remark: Note that $a_{s, i}(\rho) \neq a_{S}$, for all $i \in S \subset N$. But, given the assumption (A.3), condition (RR.2) implies that $a_{s, i}(\rho) \rightarrow a_{S}$ whenever $\rho \rightarrow 1$.

\section{NTU-games}

In this section we see that the Random marginal and the Random Removal models each support a single-valued solution in the class of NTU-games.

Random marginal. To characterize the value associated to the RM-model we need to define the concept of marginal contributions associated to a payoff configuration. Let $V$ be an NTU-game and let $\mathbf{x}=\left(x_{S}\right)_{S \subset N}$ be an efficient payoff configuration. For each coalition $S$ containing player $i$ let

$$
\partial_{\mathbf{x}}^{i}(V, S):=\max \left\{\xi^{i} \in \mathbb{R}:\left(x_{S \backslash i}, \xi^{i}\right) \in V(S)\right\}
$$

Note that if $V \in \mathcal{G}$ and $x_{S} \in V_{0}(S)$ for all $S \subset N, \partial_{\mathbf{x}}^{i}(V, S)$ is well defined and $\partial_{\mathbf{x}}^{i}(V, S) \geq 0$. One can interpret this marginal contribution as the maximum that player $i$ can get in coalition $S$ under the restriction that the others players in $S$ have at their 
disposal the outside option given by $x_{S \backslash i}$. If $V$ is a TU-game, given by the characteristic function $v$, for any efficient payoff configuration $\mathbf{x}$ it holds that $\partial_{\mathbf{x}}^{i}(V, S)=\partial^{i}(v, S)$ for all $i \in S \subset N$.

Remark. The value of the Consistent NTU-solution in Hyperplane games can also be defined recursively ${ }^{13}$ by

$$
\varphi_{S}^{i}(V)=\frac{1}{s} \partial_{\mathbf{x}}^{i}(V, S)+\sum_{j \in S \backslash i} \frac{1}{s} \varphi_{S \backslash j}^{i}(V), \quad(i \in S \subset N),
$$

where $\mathbf{x}=\left(\varphi_{S}(V)\right)_{S \subset N}$, starting with $\varphi_{\{i\}}^{i}(V)=r^{i}$, for all $i \in N$.

Because $\partial V(S)$ is a hyperplane, in the RM-model it occurs that $u_{S}(\rho) \in \partial V(S)$, so again we have that $a_{S, i}(\rho)=a_{S}(\rho)=u_{S}(\rho)$ as in Theorem (4). Applying the same arguments as there, we can reproduce for the Consistent NTU-solution in Hyperplane games the same result that as Theorem (4) yields for the Shapley value in TU-games.

We now proceed to define the NTU-value supported by the RM-model.

Definition 6 Let $V \in \mathcal{G}$ and $\mathbf{a}=\left(a_{S}\right)_{S \subset N}$ be a payoff configuration. Then $\mathbf{a}$ is the $R M$ value $\zeta$ of $V$ (i.e., $\mathbf{a}=\zeta(V)$ ) if and only if for each $S \subset N$ there exists a vector $\lambda_{S} \in \mathbb{R}_{++}^{S}$ such that:

(a) $a_{S} \in \partial V(S)$;

(b) $\lambda_{S} \cdot a_{S}=v\left(S, \lambda_{S}\right):=\max \left\{\lambda_{S} \cdot c: c \in V(S)\right\}$; and

(c) $\lambda_{S}^{i}\left(a_{S}^{i}-u_{S}^{i}\right)=\lambda_{S}^{j}\left(a_{S}^{j}-u_{S}^{j}\right)$ for all $i, j \in S$, where $u_{S}^{i}:=\frac{1}{s}\left(\partial_{\mathbf{a}}^{i}(V, S)+\sum_{k \in S \backslash i} a_{S \backslash k}^{i}\right)$ for all $i \in S$.

Condition (a) states that the payoff vector $a_{S}$ is efficient for coalition $S$. Condition (b) ensures that $a_{S}$ is also $\lambda_{S^{-}}$utilitarian, i.e., that it maximizes the sum of the $\lambda_{S^{-}}$

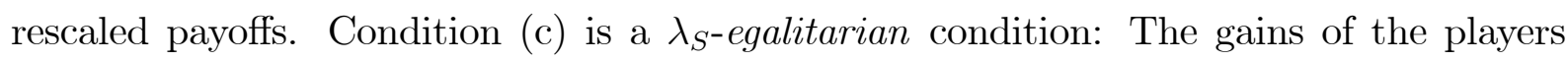

\footnotetext{
${ }^{13}$ See formula (3) in Hart and Mas-Colell (1996a).
} 
in $a_{S}$ with respect to the vector $u_{S}$ are equal relative to the units given by $\lambda_{S}$. The payoff vector $u_{S}$ has the following interpretation: The payoff allocation $\left(a_{S \backslash i}, \partial_{\mathbf{a}}^{i}(V, S)\right)$ specifies the choice of player $i \in S$ if this player has the dictatorial power to choose for $S$, under the restriction that the others players in $S$ have at their disposal $a_{S \backslash i}$. Vector $u_{S}=\frac{1}{s} \sum_{i \in S}\left(a_{S \backslash i}, \partial_{\mathbf{a}}^{i}(V, S)\right)$ gives the expected payoff allocation for players in $S$ if each member has an equal chance of obtaining this dictatorial power ${ }^{14}$. Note that condition (c) can be rewritten as

$$
\begin{aligned}
\left(c^{\prime}\right) \lambda_{S}^{i}\left[\left(a_{S}^{i}-\partial_{\mathbf{a}}^{i}(V, S)\right)+\sum_{k \in S \backslash i} \lambda_{S}^{i}\left(a_{S}^{i}-a_{S \backslash k}^{i}\right)\right]= \\
\lambda_{S}^{j}\left[\left(a_{S}^{j}-\partial_{\mathbf{a}}^{j}(V, S)\right)+\sum_{k \in S \backslash i} \lambda_{S}^{j}\left(a_{S}^{j}-a_{S \backslash k}^{j}\right)\right], \text { for all } i, j \in S .
\end{aligned}
$$

Proposition 7 If $V \in \mathcal{G}$, then the $R M$-value $\zeta$ of $V$ exists and it is unique. Moreover, if $V$ is an H-game, then $\zeta(V)$ coincides with the Consistent $N T U$-value of $V$, and, if $V$ is a $T U$-game, then $\zeta(V)$ coincides with the Shapley value of $V$.

Proof. First we prove existence and uniqueness. Let a game $V \in \mathcal{G}$. The payoff configuration $\mathbf{a}=\zeta(V)$ is built recursively as follows: We start with single coalitions $\{i\}$, for all $i \in N$, making $\lambda_{\{i\}}^{i}>0$, and $a_{\{i\}}^{i}=r^{i}$. Then conditions (a), (b), and (c), trivially hold. Assume by induction hypothesis that the Proposition holds for every $T \varsubsetneqq S$. By convexity of $V(S)$ and monotonicity, it holds that $u_{S} \in V_{0}(S)$, and the unicity of $a_{S \backslash i}$, for all $i \in S$, determine $u_{S}$ uniquely. If $u_{S} \in \partial V_{0}(S)$, making $a_{S}=u_{S}$, and taking as $\lambda_{S}$ one of the support vectors of $V(S)$ at point $a_{S}$, the Proposition holds. If $u_{S} \notin \partial V_{0}(S), \mathrm{A}$ vector $a_{S}$ satisfies (a), (b), and (c), if and only if

$$
a_{S}:=\arg \max \prod_{\substack{i \in S \\ x \geq u_{S} \\ x \in V_{0}(S)}}\left(x^{i}-u_{S}^{i}\right)
$$

\footnotetext{
${ }^{14}$ See the interpretation of the Conditional Random Dictatorship axiom used in de Clippel, Peters and Zank (2004) for the characterization of the Consistent NTU-solution.
} 
where in that case $\lambda_{S}$ must be collineal to the vector $\left(\frac{1}{a_{S}^{i}-u_{S}^{i}}\right)_{i \in S}$, and point $a_{S}$ is unique. When $V$ is an H-game, or a TU-game, it holds that all $u_{S} \in \partial V_{0}(S)$, for all $S \subset N$, and therefore $a_{S}=u_{S}$. Hence, formulas (2) and (1) apply to yield the Consistent and the Shapley values respectively.

We now establish the main result of this Section.

Theorem 8 Let $V \in \mathcal{G}$ be an NTU-game. Then for each $0 \leq \rho<1$ there is an SP equilibrium of the RM-model. Moreover, if $V$ satisfies the additional assumption (A.5), when $\rho \rightarrow 1$, every $S P$ equilibrium payoff configuration $\mathbf{a}(\boldsymbol{\rho})$ converges to $\mathbf{a}=\zeta(V)$.

\section{Proof. Existence.}

Assume that $V \in \mathcal{G}$. We prove the existence following a recursive argument. Given $0 \leq \rho<1$, let $a_{\{i\}, i}^{i}(\rho)=u_{\{i\}, i}^{i}(\rho):=r^{i} \in V_{0}(\{i\})$, for all $i \in N$. Therefore, for single coalitions, (RM.1)-(RM.4) are satisfied.

Assume that we have already determined $\left(a_{S, i}, u_{S, i}\right)$ for all $i \in S \subsetneq N$. Let $u_{N, i}(\rho)$ be defined by $u_{N, i}(\rho) \in \partial V(N)$ and $u_{N, i}^{j}(\rho):=a_{N \backslash i}^{j}(\rho)$, for all $j \neq i$, where $a_{N \backslash i}(\rho):=$ $\frac{1}{n-1} \sum_{k \in N \backslash i} a_{N \backslash i, k}(\rho)$. Because $a_{N \backslash i}(\rho) \in V_{0}(N \backslash i)$, by Monotonicity it holds that $u_{N, i}(\rho) \in$ $V_{0}(N)$, and then (RM.3) and (RM:4) are satisfied. Define $u_{N}(\rho):=\frac{1}{n} \sum_{i \in N} u_{N, i}(\rho)$, then, by Convexity, $u_{N}(\rho) \in V_{0}(N)$.

If $u_{N}(\rho) \in \partial V_{0}(N)$, making $a_{N, i}(\rho):=u_{N}(\rho)$, for all $i \in N$, conditions (RM.1) and (RM.2) are trivially satisfied.

If $u_{N}(\rho) \notin \partial V_{0}(N)$, define $V\left(u_{N}(\rho), N\right):=V_{0}(N) \cap\left(u_{N}(\rho)+\mathbb{R}_{+}^{N}\right)$. Therefore, (A.1) and (A.3) imply that $V\left(u_{N}(\rho), N\right)$ is a non-empty, compact and convex set. Define $n$ functions $\alpha_{i}(b), i \in N$, from $V\left(u_{N}(\rho), N\right)$ into itself by letting $\alpha_{i}(b)$ be defined by: $\alpha_{i}(b) \in \partial V\left(u_{N}(\rho), N\right)$ and $\alpha_{i}^{j}(b):=\rho b^{j}+(1-\rho) u_{N}^{j}(\rho)$, for all $j \neq i$. By Non-levelness and (A.1), $\alpha_{i}$ is well-defined and continuous. By convexity of $V\left(u_{N}(\rho), N\right)$, function $\alpha(\rho):=$ 
$\frac{1}{n} \sum_{i \in N} \alpha_{i}(b)$ is a continuous function that maps $V\left(u_{N}(\rho), N\right)$ into itself. Therefore, by the brower's fixed point theorem, there is a vector $a(\rho) \in V\left(u_{N}(\rho), N\right)$ satisfying $a(\rho)=\alpha(a(\rho))$. By construction, letting $a_{N, i}(\rho):=\alpha_{i}(a(\rho)), i \in N,($ RM.1) and (RM.2) are satisfied.

In this recursive way we prove the existence of payoff configuration proposals $\left(a_{S, i}(\rho), u_{S, i}(\rho)\right)_{i \in S \subset N}$ which satisfy (RM.1)-(RM.4) and, by Proposition (RM), they correspond to an SP equilibrium.

\section{Convergence.}

Consider a convergence sequence $\left\{\rho_{r}\right\} \rightarrow 1$ when $r \rightarrow \infty$. Let $\left\{\left(a_{S, i}\left(\rho_{r}\right),\left(u_{S, i}\left(\rho_{r}\right)_{i \in S \subset N}\right\} \subset\right.\right.$ $\left(\prod_{S \subset N} V_{0}(S)\right)^{2}$ be their associated SP equilibrium proposals. Because $\left(\prod_{S \subset N} V_{0}(S)\right)^{2}$ is a compact set, it must be a subsequence $\left\{\rho_{r}^{\prime}\right\}$ such that $\left\{\left(a_{S, i}\left(\rho_{r}^{\prime}\right),\left(u_{S, i}\left(\rho_{r}^{\prime}\right)_{i \in S \subset N}\right\} \rightarrow\right.\right.$ $\left\{\left(a_{S, i}\right),\left(u_{S, i}\right)_{i \in S \subset N}\right\}$. By the compactness assumption, let $(M, \ldots, M) \in \mathbb{R}_{+}^{S}$ be an upper bound of $V_{0}(S)$, then $\left|a_{S, i}^{j}(\rho)-a_{S}^{j}(\rho)\right| \leq M(1-\rho)$ for all $i, j \in S$, and all $S \subset N$. Therefore, in the limit, it holds that $a_{S, i}=a_{S}$, for all $i \in S \subset N$.

Suppose now that $V$ satisfies also the smoothness assumption (A.5). Let $\left\{\left(a_{S}\right),\left(u_{S}\right)_{S \subset N}\right\}$ be the limit payoff configuration as before, where $u_{S}=\frac{1}{s} \sum_{i \in S} u_{S, i}$, for all $S \subset N$.

First, note that $a_{\{i\}}^{i}=r^{i}$, then it trivially holds that $a_{\{i\}}=\zeta_{\{i\}}(V)$, for all $i \in N$.

Let a coalition $S \subset N$, with $s \geq 2$. Let $\pi_{S}(\rho) \in \mathbb{R}_{++}^{S}$ be defined either by an outward normal to $\partial V(S)$ if $u_{S}(\rho) \in \partial V(S)$, or by the vector $\left(\frac{1}{a_{S}^{i}(\rho)-u_{S}^{i}(\rho)}\right)_{i \in S}$ if $u_{S}(\rho) \notin \partial V(S)$ (note that in this case $a_{S, i}^{i}(\rho)>u_{S}^{i}(\rho)$ for all $i \in S$, and then $a_{S}^{i}(\rho)>u_{S}^{i}(\rho)$ ). Therefore, all $a_{S, i}^{i}(\rho), i \in S$, belong to the hyperplane $H_{S}(\rho)$ defined by

$$
H_{S}(\rho):=\left\{c \in \mathbb{R}^{S}: \pi_{S}(\rho) \cdot c=\pi_{S}(\rho) \cdot a_{S}(\rho)\right\} .
$$

To check this, if $u_{S}(\rho) \in \partial V(S)$, this holds by definition of $\pi_{S}(\rho)$. If $u_{S}(\rho) \notin \partial V(S)$, we 
have that

$$
\begin{aligned}
a_{S, i}^{i}(\rho) & =s a_{S}^{i}(\rho)-\sum_{j \in S \backslash i} a_{S, j}^{i}(\rho)=s a_{S}^{i}(\rho)-\sum_{j \in S \backslash i}\left(\rho a_{S}^{i}(\rho)-(1-\rho) u_{S}^{i}(\rho)\right) \\
& =(s-\rho(s-1))\left(a_{S}^{i}(\rho)-u_{S}^{i}(\rho)\right)+u_{S}^{i}(\rho) .
\end{aligned}
$$

hence,

$$
\begin{aligned}
\pi_{S}(\rho) \cdot a_{S, i}(\rho) & =\frac{a_{S, i}^{i}(\rho)}{a_{S}^{i}(\rho)-u_{S}^{i}(\rho)}+\sum_{j \in S \backslash i} \frac{a_{S, i}^{j}}{a_{S}^{j}(\rho)-u_{S}^{j}(\rho)} \\
& =(s-\rho(s-1))+\frac{u_{S}^{i}(\rho)}{a_{S}^{i}(\rho)-u_{S}^{i}(\rho)}+\sum_{j \in S \backslash i} \frac{\rho\left(a_{S}^{j}(\rho)-u_{S}^{j}(\rho)\right)+u_{S}^{j}(\rho)}{a_{S}^{j}(\rho)-u_{S}^{j}(\rho)} \\
& =s+\sum_{j \in S} \frac{u_{S}^{j}(\rho)}{a_{S}^{j}(\rho)-u_{S}^{j}(\rho)}=\pi_{S}(\rho) \cdot a_{S, j}(\rho), \text { for all } i, j \in S .
\end{aligned}
$$

Denote by $\pi_{S}^{*}(\rho)=\pi_{S}(\rho) \cdot \frac{1}{\sum_{i \in S} \pi_{S}^{i}(\rho)}$. Since $a_{S, i}(\rho) \rightarrow a_{S}$, by smoothness of $\partial V(S)$, we have that $\pi_{S}^{*}(\rho) \rightarrow \lambda_{S}$, where $\lambda_{S}$ is the outward unit length normal to $\partial V(S)$ at $a_{S}{ }^{15}$; $\lambda_{S}$ being collineal to the vector $\left(\frac{1}{a_{S}^{i}-u_{S}^{i}}\right)_{i \in S}$ when $u_{S} \notin \partial V(S)$. Therefore, for $\lambda_{S}$, $a_{S}$, and $u_{S}$, conditions (a), (b), and (c), of Definition (6) are satisfied.

The unicity of the limit payoff configuration $\left\{\left(a_{S}\right),\left(u_{S}\right)_{S \subset N}\right\}$ follows by a straightforward induction argument. Because $a_{\{i\}}^{i}=r^{i}$, for all $i \in N$, the breakdown payoffs $u_{S}$ are uniquely determined for all $S \subset N$, such that $s=2$. This implies the uniqueness of limit points $a_{S}$, for coalitions of size $s=2$. This fact implies again the uniqueness of limit points $u_{S}$, and hence the uniqueness of limit points $a_{S}$, for coalitions of size $s=3$, and so on and so forth up to the grand coalition $N$.

Remark. Asymmetric solutions can be easily defined. We need only assume that the proposers are chosen to be the proposer with different probabilities. Let $w \in \mathbb{R}_{++}^{N}$ be a

\footnotetext{
${ }^{15}$ Note here that for $|S \geq 3|$ the set of equilibrium offers $a_{S, i}(\rho)$ is not necessarily a singleton; and without smoothness on $\partial V(S)$, the convergence to $\zeta_{S}(V)$ may fail too. See Prooposition 8.1, and Remark 3, in Section 8, of Thomson and Lensberg (1989).
} 
vector of weights, and assume that the proposers are chosen in proportion to these weights. In particular, when $V$ is an $\mathrm{H}$-game, it holds that the asymmetric solution coincides with the weighted Shapley (1953) value for TU-games (see Kalai and Samet, 1985; and Hart and Mas-Colell, 1989), and with the weighted Consistent NTU-value for H-games (See Maschler and Owen, 1989; and Calvo, García and Zarzuelo, 2001).

Random removal. The solidarity value in NTU-games can be defined as follows.

Definition 9 Let $V \in \mathcal{G}$ and $\mathbf{a}=\left(a_{S}\right)_{S \subset N}$ be a payoff configuration. Then $\mathbf{a}$ is the Solidarity value $\psi$ of $V$ (i.e., $\mathbf{a}=\psi(V)$ ) if and only if for each $S \subset N$ there exists a vector $\lambda_{S} \in \mathbb{R}_{++}^{S}$ such that:

(a) $a_{S} \in \partial V(S)$;

(b) $\lambda_{S} \cdot a_{S}=v\left(S, \lambda_{S}\right):=\max \left\{\lambda_{S} \cdot c: c \in V(S)\right\}$; and

(d) $\lambda_{S}^{i}\left(a_{S}^{i}-d_{S}^{i}\right)=\lambda_{S}^{j}\left(a_{S}^{j}-d_{S}^{j}\right)$ for all $i, j \in S$, where $d_{S}^{i}:=\frac{1}{s} \sum_{k \in S \backslash i} a_{S \backslash k}^{i}$ for all $i \in S$.

Condition (d) is also a $\lambda_{S^{-}}$egalitarian type condition. The difference with condition (c) of the random marginal value lies in the definition of the breakdown point $d_{S}$. The vector $d_{S}=\frac{1}{s} \sum_{i \in S}\left(a_{S \backslash i}, 0\right)$ gives the expected payoff allocation for players in $S$ if each member has an equal chance of dropping out of the game, obtaining a zero payoff.

Parallel results of the random marginal approach can be obtained for the solidarity value.

Definition 10 If $V \in \mathcal{G}$, then the solidarity value $\psi$ of $V$ exists and it is unique. Moreover, if $V$ is a TU-game, then $\psi(V)$ coincides with the solidarity TU-value of $V$.

Theorem 11 Let $V \in \mathcal{G}$ be an NTU-game. Then for each $0 \leq \rho<1$ there is an $S P$ equilibrium of the RR-model. Moreover, if $V$ satisfies the additional assumption (A.5), 
when $\rho \rightarrow 1$, every $S P$ equilibrium payoff configuration $\mathbf{a}(\boldsymbol{\rho})$ converges to $\mathbf{a}=\psi(V)$.

The proofs are fully identical to the RM-model, interchanging the roles of $u_{S}$ by $d_{S}$, hence they are left to the reader.

Remark. The solidarity value has an interesting link with the equal split solution, relative to $(0, \ldots, 0)$. In Hart and Mas-Colell $(1996 \mathrm{a})$ this solution is obtained as one of possible variations of the breakdown technology. In particular, as they point out in Case (b): Only the responders (but not the proposer) drop out, all with equal probability. This solution can be defined in NTU-games as follows.

Definition 12 Let $V \in \mathcal{G}$ and $\mathbf{a}=\left(a_{S}\right)_{S \subset N}$ be a payoff configuration. Then $\mathbf{a}$ is the equal split value $\phi$ of $V$ (i.e., $\mathbf{a}=\phi(V)$ ) if and only if for each $S \subset N$ there exists a vector $\lambda_{S} \in \mathbb{R}_{++}^{S}$ such that:

(a) $a_{S} \in \partial V(S)$;

(b) $\lambda_{S} \cdot a_{S}=v\left(S, \lambda_{S}\right):=\max \left\{\lambda_{S} \cdot c: c \in V(S)\right\}$; and

(e) $\lambda_{S}^{i} a_{S}^{i}=\lambda_{S}^{j} a_{S}^{j}$ for all $i, j \in S$.

Note that if $V$ is a TU-game, then $a_{S}^{i}=\frac{v(S)}{s}$, for all $i \in S \subset N$. Hart and Mas-Collel mention that, in TU-games, the solidarity value approaches, for a large $n$, the equal split value. We will confirm this assertion for large NTU-games in the next Section.

Remark. The properties of Uniqueness and Symmetry are the main motivation of the paper. By Uniqueness we mean that the payoff configuration of the solution is single-valued. This property is important if we consider a solution as a way to find an agreement when an alternative must be chosen over a set that produces a different rank of preferences. This lack of unanimity is not solved when a solution selects a subset of alternatives, because we again have the same ranking problem among players, but now over the subset selected. The example of Section 2 shows that neither the Shapley, 
Harsanyi and Consistent NTU-values satisfy this requirement ${ }^{16}$.

A TU-game $(N, v)$ is said to be symmetric when the worth of a coalition is a function of its size, i.e. $v(s)=f(|S|)$. A Pure Bargaining game $(d, V(N))$ is symmetric if $d_{1}=$ $d_{2}=\ldots=d_{n}$, and for any bijection $\pi: N \rightarrow N$, and for each $x \in V(N)$ it holds that $\pi x \in V(N)$, where $\pi x=\left(x_{\pi(i)}\right)_{i \in N}$. We extend the definition of Symmetry for NTUgames as follows: $(N, V)$ is symmetric if $(i) r^{i}=r^{j}$ for all $i, j \in N$, and (ii) for any two $S, T \subset N$, such that $|S|=|T| \geq 2$, for any bijection $\pi: S \rightarrow T$, and any $x \in V(S)$, it holds that $\pi x \in V(T)$ (note that the case $S=T$ is included). We say that a solution satisfies Symmetry if it selects symmetric payoffs when the game $V$ is symmetric, i.e. for every $\mathbf{x} \in \Psi(V), x_{S}^{i}=x_{S}^{j}$ for all $i, j \in S \subset N$. Because the Nash solution satisfies symmetry for Pure Bargaining games, and given the way in which the random marginal and the solidarity NTU-values are built, it can be checked that they also satisfy Symmetry in $\mathcal{G}$.

A full axiomatic characterization of these new solutions is not yet accomplished, and should be the object of further research.

\section{$7 \quad$ Large games}

The aim of this Section is a study of how the random marginal and the solidarity values should behave in large games with non-transferable utility. More specifically, we will consider only differentiable market games for which the Value equivalence theorem holds (Aumann, 1975); that is, the equivalence between the value allocations and the core allocations. The basic references and results in this topic can be found in Hart (1994, and 2001).

\footnotetext{
${ }^{16}$ For the Consistent NTU-value a three-person example of non unicity can be found in Owen (1994).
} 
We restrict our analysis to the case of continuum games with finitely many types of players, where each coalition is characterized by its composition. Let $N=\{1, \ldots, n\}$ be the set of types. The profile of a coalition is a vector $x \in \mathbb{R}_{+}^{N}$, where $x^{i}$ is the mass of players of type $i$ in the coalition. The game form will specify the sets of feasible payoff vectors for all coalitions. We consider only type-symmetric imputations, where all players of the same type get the same payoff ${ }^{17}$. For every $x \in \mathbb{R}_{+}^{N}$, let $V(x) \subset$ $\mathbb{R}^{N}$ be the set of feasible per-capita payoff vectors for a coalition with profile $x$. This point-to-set map $V$ is called the $N T U$-game form. Given $V$ we define also the set of feasible total per-type payoffs by $\hat{V}(x):=\{x * a: a \in V(x)\}$, for all $x \in \mathbb{R}_{+}^{N}$. Define $\hat{v}(x, \lambda):=\sup \{\lambda \cdot(a * x): a \in V(x)\} \equiv \sup \{\lambda \cdot b: b \in \hat{V}(x)\} \cdot \hat{v}(x, \lambda)$ is the continuum TU-game associated to $V$ with utility comparison weights $\lambda$. We will make the following assumptions:

(C.1) Standard: For every $x \in \mathbb{R}_{+}^{N}, V(x)$ is a non-empty and strict subset of $\mathbb{R}^{N}$, it is closed, convex, comprehensive and non-level. $V_{0}(x):=V(x) \cap \mathbb{R}_{+}^{N}$ is non-empty and bounded.

(C.2) Super-additivity: $\hat{V}(x)+\hat{V}(y) \subset \hat{V}(x+y)$ for every $x, y \in \mathbb{R}_{+}^{N}$.

(C.3) Homogeneity: $V(t x)=V(x)$ for every $x \in \mathbb{R}_{+}^{N}$ and $t>0$ (in terms of total payoffs, $\hat{V}(t x)=t \hat{V}(x))$.

(C.4) Differentiability: For any $\lambda \in \mathbb{R}_{++}^{N}$, the gradient $\nabla_{x} \hat{v}(\bar{x}, \lambda)$ exists for all $\bar{x} \in \mathbb{R}_{++}^{N}$. $\nabla_{x} \hat{v}(\bar{x}, \lambda)$ is uniformly bounded and uniformly positive for every bounded subset of $\mathbb{R}_{++}^{N}$. Moreover $\hat{v}(x, \lambda)$ is $C^{2}$ on its domain.

Assumptions C.1, C.2, and C.3 are standard for market games. For example, a pure exchange economy where each type $i$ possesses a utility function $u_{i}$ concave and non-

\footnotetext{
${ }^{17}$ Because the random marginal value yields the same payoffs to substitute players, this is not a real restriction.
} 
decreasing, with the slope everywhere bounded away from 0 and infinity, and $u_{i}\left(\omega_{i}\right)=0$ (where $\omega_{i}$ is the initial endowment), yields a market game satisfying C.1-C.3. Differentiability allows us to set the condition that characterizes the RM-value in terms of a first order partial differential equations system.

Remark. Note that the differentiability of $\hat{v}(x, \lambda)$ implies both the smoothness of $\partial V(x)$, and the strict convexity of $V(x)$. Therefore there is a unique $a \in V(x)$ such that $\lambda \cdot(a * x)=\hat{v}(x, \lambda)$. Conversely, for any $a \in \partial V(x)$ there is a unique $\lambda$, such that $\lambda \cdot(a * x) \geq \lambda \cdot(b * x)$, for all $b \in V(x)$. That is, there is a unique supporting hyperplane to $\hat{V}(x)$ at $a * x, \lambda$ being its corresponding outward normal vector.

Random marginal. We now see how the conditions to be a random marginal value will look for large games. As in Hart and Mas-Colell (1996b), given a continuum game $V$ and the grand coalition $x \in \mathbb{R}_{++}^{N}$, we obtain a sequence of finite games $V_{r}$, for $r=1,2, \ldots$, with the set of players $N_{r}:=\left\{i_{1}, \ldots, i_{r}\right\}_{i \in N}$; where there is a set of types $N$, having for each type $i \in N, r$ symmetric players, each one being regarded as having a mass of $\frac{\bar{x}^{i}}{r}$. There are in total $\# N_{r}=r n$ players. For any coalition $S \subset N_{r}$, denote by $m(S):=\left(m^{i}(S)\right)_{i \in N}$, where $m^{i}(S)$ is the number of players of type $i$ in $S$. Let $V_{r}$ be the set of feasible per-capita typesymmetric payoff vectors; then, for any $S \subset N_{r}, V_{r}(S):=V\left(m(S) *\left(\frac{1}{r} \cdot x\right)\right)$. In terms of total payoffs, $\hat{V}_{r}(S):=m(S) *\left(\frac{1}{r} \cdot x\right) * V\left(m(S) *\left(\frac{1}{r} \cdot x\right)\right)$. In particular, for all $r$, it holds that $V_{r}\left(N_{r}\right)=V(x)$, and $\hat{V}_{r}\left(N_{r}\right)=x * V(x)=\hat{V}(x)$. We say that $V_{r}$ is the $r$-approximation to the continuum game $V$. For any $S \subset N_{r}$ denote by $I(S):=\left\{i \in N: m^{i}(S) \neq 0\right\}$. A percapita type-symmetric payoff configuration is a family $\mathbf{a}_{r}=\left(a_{r, S}^{i}\right)_{i \in I(S) ; S \subset N_{r}}$, where each of these $m^{i}(S)$ players of type $i$ in coalition $S$, receives $a_{r, S}^{i_{k}}=a_{r, S}^{i}$, for all $k=1, \ldots, m^{i}(S)$.

Our assumptions imply that each finite game $\hat{V}_{r}$ has a unique per-capita random marginal value $\mathbf{a}_{r}=\zeta\left(V_{r}\right)$, and for every $S \subset N_{r}$ it holds that $a_{r, S} \in \partial V_{r}(S) \cap \mathbb{R}_{+}^{S}$. Given a continuum game $V$ and coalition $x \in \mathbb{R}_{++}^{N}$ define the per capita asymptotic random 
marginal value by $a(x):=\lim _{r \rightarrow \infty} a_{r, N_{r}}$, where $a_{r, N_{r}}$ is the per capita RM-value for the grand coalition $N_{r}$ of the finite game $V_{r}^{18}$. Because $\partial V_{r}(N) \cap \mathbb{R}_{+}^{S}=\partial V(x) \cap \mathbb{R}_{+}^{S}$ is a compact set, the existence of such limit points is a guarantee (taken the appropriate subsequence). Nevertheless, given the uniqueness of $a_{r, N_{r}}$ for every $r$, we have the plausible conjecture that the set of limit points is single valued, and the family of continuum games in which the value is well defined is still a conjecture.

A very relevant consequence of the uniqueness of the RM-value for each finite approximation $V_{r}$ is that if the continuum game $V$ is homogeneous then the asymptotic RM-value, if it does converge, must also be single-valued and homogeneous. On the contrary, denote by $a(x)$ the asymptotic RM-value for the grand coalition $x$, and suppose that for two different $t, t^{\prime}>0$, it holds that $a(t x) \neq a\left(t^{\prime} x\right)$. By homogeneity, $V(x)=V(t x)=V\left(t^{\prime} x\right)$, therefore $a(t x)$ and $a\left(t^{\prime} x\right)$ must also be solutions for $V$ at $x$. Then, for a large enough $\bar{r}$, and for all $r \geq \bar{r}$, there must be $a_{r, N_{r}}$ close to $a(t x)$, and $a_{r, N_{r}}^{\prime}$ close to $a\left(t^{\prime} x\right)$, with $a_{r, N_{r}} \neq a_{r, N_{r}}^{\prime}$, which contradicts the uniqueness of the RM-value for finite games.

Let us see the conditions that the per capita RM-value should satisfy on continuum games. Firstly, for each finite $r$-approximation $V_{r}$ and coalition $S=N_{r}$, there exists $\lambda_{r, N_{r}} \in \mathbb{R}_{++}^{N}$ such that:

(a) $a_{r, N_{r}} \in \partial V_{r}\left(N_{r}\right)$

(b) $\sum_{i \in N} \lambda_{r, N}^{i} x^{i} a_{r, N_{r}}^{i}=\max \left\{\sum_{i \in N} \lambda_{r, N}^{i} x^{i} b^{i}: b \in V_{r}\left(N_{r}\right)\right\}$;

(c') $\lambda_{r, N}^{i}\left[\left(a_{r, N_{r}}^{i}-\partial_{\mathbf{a}_{r}}^{i}\left(V_{r}, N_{r}\right)+(r-1)\left(a_{r, N_{r}}^{i}-a_{r, N_{r} \backslash i_{r}}^{i}\right)+\sum_{k \in N \backslash i} r\left(a_{r, N_{r}}^{i}-a_{r, N_{r} \backslash k_{r}}^{i}\right)\right)\right]=$ $\lambda_{r, N}^{j}\left[\left(a_{r, N_{r}}^{j}-\partial_{\mathbf{a}_{r}}^{j}\left(V_{r}, N_{r}\right)\right)+(r-1)\left(a_{r, N_{r}}^{j}-a_{r, N_{r} \backslash j_{r}}^{j}\right)+\sum_{k \in N \backslash j} r\left(a_{r, N_{r}}^{j}-a_{r, N_{r} \backslash k_{r}}^{j}\right)\right]$. $(i, j \in N) \cdot \lambda^{i}$

\footnotetext{
${ }^{18}$ More general convergence sequences of $r$-approximations could be defined, for example, by allowing different $r_{i}$ 's for different $i$ 's. But even for this symmetric definition, the limit approach is hard enough.
} 
Where

$$
\partial_{\mathbf{a}_{r}}^{i}\left(V_{r}, N_{r}\right):=\max \left\{\xi^{i} \in \mathbb{R}:\left(a_{r, N_{r} \backslash i_{r}}, \xi^{i}\right) \in V_{r}\left(N_{r}\right)\right\}, \quad(i \in N) .
$$

Taken limits, when $r \rightarrow \infty$, the efficient condition $a_{r, N_{r}} \in \partial V_{r}\left(N_{r}\right)$ turns into condition (i) $a(x) \in \partial V(x)$.

The $\lambda$-utilitarian condition (b) takes the form

(ii) $\sum_{i \in N} \lambda^{i}(x) x^{i} a^{i}(x)=\max \left\{\sum_{i \in N} \lambda^{i}(x) x^{i} b^{i}: b \in V(x)\right\}=\hat{v}(x, \lambda(x))$.

Note that, under the differentiability assumption, the $\lambda(x)$ associated to $a(x)$ is unique (up to length normalization).

For the $\lambda$-egalitarian condition $\left(c^{\prime}\right)$, the increments

$$
(r-1)\left(a_{r, N_{r}}^{i}-a_{r, N_{r} \backslash i_{r}}^{i}\right)+\sum_{k \in N \backslash i} r\left(a_{r, N_{r}}^{i}-a_{r, N_{r} \backslash k_{r}}^{i}\right)
$$

are replaced by derivatives, so they take the form

$$
x^{i} \frac{\partial a^{i}(x)}{\partial x^{i}}+\sum_{k \in N \backslash i} x^{k} \frac{\partial a^{i}(x)}{\partial x^{k}} .
$$

Define $\xi_{i, N_{r}}:=\left(a_{r, N_{r} \backslash k_{r}}, \partial_{\mathbf{a}_{r}}^{k}\left(V_{r}, N_{r}\right)\right)$; then, by construction, $\xi_{i, N_{r}} \in \partial V_{r}\left(N_{r}\right)=\partial V(x)$. Let $\lambda\left(\xi_{i, N_{r}}\right)$ be its unique associated outward normal vector. Then it holds that

$$
\lambda^{i}\left(\xi_{i, N_{r}}\right) x^{i} \partial_{\mathbf{a}_{r}}^{k}\left(V_{r}, N_{r}\right)+\sum_{k \in N \backslash i} \lambda^{k}\left(\xi_{i, N_{r}}\right) x^{k} a_{r, N_{r} \backslash i_{r}}^{k}=\hat{v}\left(x, \lambda\left(\xi_{i, N_{r}}\right)\right) .
$$

When $r \rightarrow \infty, \xi_{i, N_{r}} \rightarrow a(x)$ and $\lambda\left(\xi_{i, N_{r}}\right) \rightarrow \lambda(x)$. and hence

$$
\partial_{\mathbf{a}_{r}}^{k}\left(V_{r}, N_{r}\right) \rightarrow \frac{1}{\lambda^{i}(x)} \frac{\partial \hat{v}(x, \lambda(x))}{\partial x^{i}} .
$$

All together they suggest the following definition: 
Definition 13 Let $V$ be a continuum game with a finite type of players $N$, satisfying (C.1), (C.2), and (C.4). Let a $(x)$ be a $C^{1}$ per capita payoff configuration, $x \in \mathbb{R}_{++}^{N}$. Then $a(x)$ is a continuum RM-value of $V$ at $x$, if there exists $\lambda(x) \in \mathbb{R}_{++}^{N}$ such that

(i) $a(x) \in \partial V(x)$;

(ii) $\lambda(x) \cdot(x * a(x))=\hat{v}(x, \lambda(x))$; and

(iii) $\lambda^{i}(x)\left(a^{i}(x)-\frac{1}{\lambda^{i}(x)} \frac{\partial \hat{v}(x, \lambda(x))}{\partial x^{i}}+\sum_{k \in N} x^{k} \frac{\partial a^{i}(x)}{\partial x^{k}}\right)=\lambda^{j}(x)\left(a^{j}(x)-\frac{1}{\lambda^{j}(x)} \frac{\partial \hat{v}(x, \lambda(x))}{\partial x^{j}}+\sum_{k \in N} x^{k} \frac{\partial a^{j}(x)}{\partial x^{k}}\right)$, for all $i, j \in N$.

Condition (iii) is a system of first order partial differential equations, and if the asymptotic RM-value does exist, then it must be a particular solution of (i)-(iii). We now see the relationship between the asymptotic RM-value and the Shapley NTU-value in continuum games. The construction of a Shapley NTU-value in continuum games is as follows (see Shapley, 1969; and Shapley and Shubik, 1969).

Given a continuum game $V$, and a vector of weights $\lambda \in \mathbb{R}_{++}^{N}$, build the continuum TU-game $\hat{v}(x, \lambda)$. Under differentiability, the Aumann-Shapley (1974) value of $\hat{v}(x, \lambda)$ is defined by

$$
\varphi^{i}(\hat{v}(x, \lambda))=\int_{0}^{1} \frac{\partial \hat{v}(t x, \lambda)}{\partial x^{i}} d t, \quad \text { for all } i \in N .
$$

Definition 14 Let $V$ be a continuum game with a finite type of players $N$, satisfying (C.1), (C.2), and (C.4). Let $a(x)$ be a per capita payoff configuration, $x \in \mathbb{R}_{++}^{N}$. Then $a(x)$ is a continuum Shapley NTU-value of $V$ at $x$, if there exists $\lambda(x) \in \mathbb{R}_{++}^{N}$ such that (i) $a(x) \in \partial V(x)$; and (iv) $\lambda(x) * a(x)=\varphi(\hat{v}(x, \lambda(x)))$.

Assuming homogeneity we have the following result:

Theorem 15 Let $V$ be a continuum game with a finite type of players $N$, satisfying (C.1), (C.2), (C.3), and (C.4). Let $a(x)$ be an homogeneous $C^{1}$ per-capita payoff configuration, 
$x \in \mathbb{R}_{++}^{N}$. Then $a(x)$ is a continuum RM-value if and only if it is a Shapley NTU-value of $V$ at $x$.

Proof. Let $a(x)$ be a continuum MR-value of $V$ at $x$, and assume that it is homogenous. Because $V(x)$ is homogenous of degree $0, a(x)$ also has the same degree of homogeneity. Therefore, by Euler's formula, $\sum_{k \in N} x^{k} \frac{\partial a^{i}(x)}{\partial x^{k}}=0$, and hence condition (iii) is equivalent to

$$
\lambda^{i}(x) a^{i}(x)-\frac{\partial \hat{v}(x, \lambda(x))}{\partial x^{i}}=k ; \text { for all } i \in N,
$$

where $k \in \mathbb{R}$. By multiplying this expression by $x^{i}$, and adding it over all $i$, we have

$$
\sum_{i \in N} \lambda^{i}(x) x^{i} a^{i}(x)-\sum_{i \in N} x^{i} \frac{\partial \hat{v}(x, \lambda(x))}{\partial x^{i}}=\left(\sum_{i \in N} x^{i}\right) k .
$$

By condition (ii), $\sum_{i \in N} \lambda^{i}(x) x^{i} a^{i}(x)=\hat{v}(x, \lambda(x))$. And because $\hat{v}(x, \lambda(x))$ is homogeneous of degree 1, by applying Euler's formula again, $\sum_{i \in N} x^{i} \frac{\partial \hat{v}(x, \lambda(x))}{\partial x^{i}}=\hat{v}(x, \lambda(x))$. As $\sum_{i \in N} x^{i}>0$, it follows that $k=0$, which implies that

$$
\lambda^{i}(x) a^{i}(x)=\frac{\partial \hat{v}(x, \lambda(x))}{\partial x^{i}}, \text { for all } i \in N .
$$

On the other hand, under homogeneity,

$$
\varphi(\hat{v}(x, \lambda(x)))=\int_{0}^{1} \frac{\partial \hat{v}(t x, \lambda(x))}{\partial x^{i}} d t=\frac{\partial \hat{v}(x, \lambda(x))}{\partial x^{i}}, \text { for all } i \in N,
$$

which finally yields

$$
\lambda(x) * a(x)=\nabla_{x} \hat{v}(x, \lambda(x))=\varphi(\hat{v}(x, \lambda(x))) .
$$

Because in homogenous and differentiable games the Shapley NTU-value is a homogenous and differentiable mapping, it turns out that it satisfies conditions (i)-(iii), hence both values coincide. 
If the continuum game $V$ is non-homogeneous, the RM-value and the Shapley NTUvalue will generally yield different payoffs. An alternative way to compare both values is to consider the equivalent of condition (iii) for the Shapley NTU-value. Recall for finite games its characterization:

Definition 16 Let $V \in \mathcal{G}$ and $\mathbf{a}=\left(a_{S}\right)_{S \subset N}$ be a payoff configuration. Then $a_{N}$ is the Shapley NTU-value $\varphi$ of $V$ if and only if for each $S \subset N$ there exists a vector $\lambda_{S} \in \mathbb{R}_{++}^{S}$ such that:

$\left(a^{\prime}\right) a_{N} \in \partial V(N)$;

(b) $\lambda_{S} \cdot a_{S}=v\left(S, \lambda_{S}\right)$; and

(f) $\lambda_{S}^{i}\left(a_{S}^{i}-a_{S \backslash j}^{i}\right)=\lambda_{S}^{j}\left(a_{S}^{j}-a_{S \backslash i}^{j}\right)$ for all $i, j \in S$.

Note that, in the payoff configuration a, efficiency (and hence, feasibility) is only required for the grand coalition $N$. Condition (f) is the $\lambda$-Balanced Contributions property. The main drawback of this definition is given by the possible non-feasibility of the threat points $a_{S}$, for all $S \neq N$ (except in the TU-case).

By using similar arguments as above, in the continuum, conditions ( $\left.a^{\prime}\right)$ and (b) turn into (i) and (ii); and condition (f) into

(iv) $\lambda^{i}(x) \frac{\partial a^{i}(x)}{\partial x^{j}}=\lambda^{j}(x) \frac{\partial a^{j}(x)}{\partial x^{i}}$, for all $i, j \in N$.

Conditions (iii) and (iv) can also be compared with condition

(v) $\sum_{j \in N} \lambda^{i}(x) x^{j} \frac{\partial a^{i}(x)}{\partial x^{j}}=\sum_{j \in N} \lambda^{j}(x) x^{j} \frac{\partial a^{j}(x)}{\partial x i}$, for all $i \in N$.

This condition, jointly with (i) and (ii), characterizes the Consistent NTU-values in continuum games (see Owen, 1996, and Leviatan, 2002).

Random removal. The condition (d) for the solidarity value is equivalent to (d') $\lambda_{S}^{i}\left(a_{S}^{i}+\sum_{k \in S \backslash i}\left(a_{S}^{i}-a_{S \backslash k}^{i}\right)\right)=\lambda_{S}^{j}\left(a_{S}^{j}+\sum_{k \in S \backslash i}\left(a_{S}^{j}-a_{S \backslash k}^{j}\right)\right)$, for all $i, j \in S$. 
Therefore, by using similar arguments as for the RM-value, we can define the solidarity value in continuum games as follows:

Definition 17 Let $V$ be a continuum game with a finite type of players $N$, satisfying (C.1), (C.2), and (C.4). Let a(x) be a $C^{1}$ per capita payoff configuration, $x \in \mathbb{R}_{++}^{N}$. Then $a(x)$ is a continuum solidarity value of $V$ at $x$, if there exists $\lambda(x) \in \mathbb{R}_{++}^{N}$ such that (i) $a(x) \in \partial V(x)$;

(ii) $\lambda(x) \cdot(x * a(x))=\hat{v}(x, \lambda(x))$; and

(iii) $\lambda^{i}(x)\left(a^{i}(x)+\sum_{k \in N} x^{k} \frac{\partial a^{i}(x)}{\partial x^{k}}\right)=\lambda^{j}(x)\left(a^{j}(x)+\sum_{k \in N} x^{k} \frac{\partial a^{j}(x)}{\partial x^{k}}\right)$, for all $i, j \in N$.

Given the uniqueness of the solidarity value for finite games, we can also conjecture that the asymptotic solidarity value exists and it is single valued. Moreover, in homogeneous games it must also be a homogeneous value. Hence it implies that, under differentiability, $\sum_{k \in N} x^{k} \frac{\partial a^{j}(x)}{\partial x^{k}}=0$. For this reason, condition (iii) turns into condition

(vi) $\lambda^{i}(x) a^{i}(x)=\lambda^{j}(x) a^{j}(x)$, for all $i, j \in N$,

which, jointly with conditions (i) and (ii), characterize the equal split solution for continuum NTU-games. So, in homogeneous games, both values coincide.

\section{References}

Aumann, R.J. (1975): "Values of Markets with a Continuum of Traders," Econometrica $43,611-646$.

(1985): “An Axiomatization of the Non-Transferable Utility Value," Econometrica 53, 599-612.

Aumann, R.J., And L.S. Shapley (1974): Values of Non-atomic Games. Princeton University Press. 
Calvo, E., I. Garcia, and J.M. Zarzuelo (2001): "Replication Invariance on NTU games," International Journal of Game Theory 29, 473-486.

de Clippel, J., H. Peters, and H. Zank (2004): "Axiomatizing the Harsanyi Solution, the Symmetric Egalitarian Solution, and the Consistent Shapley Solution for NTU-games," International Journal of Game Theory 33, 145-158.

GuL, F. (1989): "Bargaining Fundations of Shapley Value," Econometrica 57, 81-95.

Harsanyi, J.C. (1963): "A Simplified Bargaining Model for the n-Person Cooperative Game," International Economic Review 4, 194-220.

Hart, O., And J. Moore (1990): "Property Rigts and the Nature of the Firm," Journal of Political Economy 98, 1119-1158.

Hart, S. (1985): "An Axiomatization of Harsanyi's Nontransferable Utility Solution," Econometrica 53, 1295-1313.

(1994): “On Prize Games," in Essays in Game Theory, ed. by N. Meggido. Springer-Verlag, New York, 111-121.

(2001): "Values of perfectly Competitive Economies," in Handbook of Game Theory, with Economic Aplications, vol. III, ed. by R.J. Aumann and S. Hart. North-Holland.

_ (2005): "An Axiomatization of the Consistent Non-Transferable Utility Value," International Journal of Game Theory 33, 355-366 .

Hart, S., And A. Mas-Colell (1989): "Potential, Value, and Consistency," Econometrica 57, 589-614. 
(1996a): "Bargaining and Value," Econometrica 64, 357-380.

(1996b): "Harsanyi Values of Large economies: Nonequivalence to Competitive Equilibria," Games and Economic Behavior 13, 74-99.

Kalai, E., And D. SAmet (1987): "On Weighted Shapley Values," International Journal of Game Theory 16, 205-222.

Kalai, E., And M. Smorodinsky (1975): “Other solutions to Nash's bargaining problem," Econometrica 43, 513-518.

Leviatan, S. (2002): "Consistent values and the core in continuum market games with two type of players," International Journal of Game Theory 31, 383-410.

Maschler, M., And G. Owen (1989): “The Consistent Shapley Value for Hyperplane Games," International Journal of Game Theory 18, 389-407.

_ (1992): "The Consistent Shapley Value for Games without Side Payments," in Rational Interaction, ed. by R. Selten. Springer-Verlag, New York, 5-12.

Myerson, R.B. (1980): "Conference Structures and Fair Allocation Rules," International Journal of Game Theory 9, 169-182.

NASH, J.F. (1950): “The bargaining problem," Econometrica 18, 155-162.

NowAK, A.S., AND T. RADZIK (1994): "A solidarity value for $n$-person transferable utility games," International Journal of Game Theory 23, 43-48.

Owen, G. (1994): "The Non-Consistency and Non-Uniqueness of the Consistent Value," in Essays in Game Theory, ed. by N. Megiddo. Springer-Verlag, New York, 155-162. 
(1996): "The Consistent Solution for Non-Atomic Games," in Understanding Strategic Interaction: Essays in Honor of Reinhard Selten, ed. by W. Albers, W. Guth, P. Hammerstien, B. Moldovanu and E. Van Damme. Berlin (Springer), 279285.

Perez-Castrillo, D., And D. Wettstein (2001): "Bidding for the Surplus: A Noncooperative Approach to the Shapley Value," Journal of Economic Theory 100, 274-294.

Shapley, L.S. (1953): "A Value for n-Person Games," in Contributions to the theory of Games II (Annals of Mathematics Studies 28), ed. by H. W. Kuhn, and A. W. Tucker. Princeton University Press, Princeton, 307-317.

- (1969): "Utility comparison and the theory of games," in La Décision, ed. by G.Th. Guilbaud. Editions du CNRS, Paris.

Shapley, L.S., And M. Shubik (1969): "Pure Competition, Coalition Power and Fair Division," International Economic Review 69, 337-362.

Thomson, W., And T. Lensberg (1989): Axiomatic Theory of Bargaining with a Variable Number of Agents. Cambridge: Cambridge University Press.

Vidal-Puga, J.J. (2006): "Forming coalitions and the Shapley NTU value," Mimeo.

Winter, E. (1994): "The Demand Commitment Bargaining and Snowballing Cooperation," Economic Theory 4, 225-273. 